\title{
TIME REVERSAL OF SOME STATIONARY JUMP DIFFUSION PROCESSES FROM POPULATION GENETICS
}

\author{
MARTIN HUTZENTHALER, ${ }^{*}$ Goethe-University Frankfurt \\ JESSE EARL TAYLOR, ${ }^{* *}$ Arizona State University
}

\begin{abstract}
We describe the processes obtained by time reversal of a class of stationary jump diffusion processes that model the dynamics of genetic variation in populations subject to repeated bottlenecks. Assuming that only one lineage survives each bottleneck, the forward process is a diffusion on $[0,1]$ that jumps to the boundary before diffusing back into the interior. We show that the behavior of the time-reversed process depends on whether the boundaries are accessible to the diffusive motion of the forward process. If a boundary point is inaccessible to the forward diffusion then time reversal leads to a jump diffusion that jumps immediately into the interior whenever it arrives at that point. If, instead, a boundary point is accessible then the jumps off of that point are governed by a weighted local time of the time-reversed process.
\end{abstract}

Keywords: Time reversal; jump diffusion; local time; coalescent; population bottleneck; selective sweep

2010 Mathematics Subject Classification: Primary 60J60

Secondary 60J55; 92D10

\section{Introduction}

Kingman's observation that the genealogy of a random sample of individuals from a panmictic, neutrally evolving population can be represented as a Markov process [16], [17] ranks as one of the most influential contributions to mathematical population genetics. Not only has the coalescent led to a deeper understanding of evolution in neutral populations, but it also plays a central role in statistical genetics where it facilitates the efficient simulation of sample genealogies. Unfortunately, the Markov property that makes Kingman's coalescent both mathematically and computationally tractable is usually not shared by genealogical processes in populations composed of nonexchangeable individuals. In particular, this is true when there are fitness differences between individuals, since then the selective interactions between individuals cause genealogies to depend on the history of lineages that are nonancestral to the sample. The key to overcoming this difficulty is to extend the genealogy to a higher-dimensional process that does satisfy the Markov property. This has been done in two ways. One approach is to embed the genealogical tree within a graphical process called the ancestral selection graph [6], [18],

Received 21 September 2009; revision received 24 June 2010.

* Current address: LMU Biozentrum, Grosshadern Str. 2, D-82152 Planegg-Martinsried, Germany.

Email address: hutzenthaler@bio.lmu.de

Research supported by the DFG in the Dutch German Bilateral Research Group Mathematics of Random Spatial Models from Physics and Biology (For 498).

** Postal address: School of Mathematical and Statistical Sciences, Arizona State University, PO Box 871804, Tempe, AZ 85287-1804, USA.

Research supported by the EPSRC, grant no. GR/T19537/01. 
[24] in which lineages can both branch and coalesce. The intuition behind this construction is that the effects of selection on the genealogy can be accounted for by keeping track of a pool of potential ancestors which includes lineages that have failed to persist due to being out-competed by individuals of higher fitness.

An alternative approach was proposed by Kaplan et al. [12], who showed that the genealogical history of a sample of genes under selection can be represented as a structured coalescent process. Here we think of the population as being divided into several panmictic subpopulations (called genetic backgrounds) which consist of individuals that share the same genotype at the selected locus. Because individuals with the same genotype are exchangeable (i.e. they have the same fitness), the rate of coalescence within a background depends only on the size of the background and the number of ancestral lineages sharing that genotype. Thus, to obtain a Markov process, we need to keep track of two kinds of information: (i) the type of ancestral lineages, and (ii) the frequencies of the alleles segregating at the selected locus, followed backwards in time. For many applications, it is assumed that the population is at equilibrium and that the forwards-in-time dynamics of the allele frequencies are described by a stationary diffusion process. In this case, the ancestral process of allele frequencies can be identified by time reversal of the diffusion process. In particular, if the diffusion process is one-dimensional then the time-reversed process conveniently has the same law as the forward process. A formal derivation of the structured coalescent process for such an equilibrium population is given in [2] and various applications are discussed in [1], [5], and [30].

The focus of this paper is on the time reversal of a population genetical model that incorporates mutation, selection, genetic drift, and population bottlenecks. To be concrete, consider a locus with two alleles, $A_{0}$ and $A_{1}$, and let $p^{N}(t)$ denote the frequency of $A_{1}$ at time $t$ in a population of size $N$. In the absence of bottlenecks, we will suppose that the jump process $p^{N}(\cdot)$ can be approximated by the Wright-Fisher diffusion $p(\cdot)$ with generator

$$
\begin{aligned}
A \phi(p) & =\frac{1}{2} p(1-p) \phi^{\prime \prime}(p)+\left(\mu_{0}(1-p)-\mu_{1} p+s(p) p(1-p)\right) \phi^{\prime}(p) \\
& \equiv \frac{1}{2} v(p) \phi^{\prime \prime}(p)+\mu(p) \phi^{\prime}(p),
\end{aligned}
$$

where $\mu_{0}$ and $\mu_{1}$ are the scaled mutation rates from $A_{0}$ to $A_{1}$ and from $A_{1}$ to $A_{0}$, respectively, and $s(p)$ is the scaled and possibly frequency-dependent selection coefficient of $A_{1}$ relative to $A_{0}$. In using the diffusion approximation, we assume that $N$ is large, that time is measured in units of $N$ generations, and that the unscaled mutation rates and selection coefficient are of order $N^{-1}$. Convergence results justifying the passage to the diffusion limit can be found in [7].

Population bottlenecks are transient events during which most of the population is descended from a small number of individuals. On the diffusive time scale, these can be modeled as instantaneous jumps in the allele frequencies, and in this paper we will be concerned with a class of models in which the bottlenecks always result in the temporary fixation of one of the two alleles, i.e. $p(\cdot)$ always jumps to 0 or 1 . We have two scenarios in mind. In the first, we consider a locus that is part of a nonrecombining segment of DNA (e.g. a mammalian mitochondrial genome) subject to strong selective sweeps which occur at rate $\lambda$. During each sweep, a unique copy of a favorable mutation arises at some linked site and rises rapidly to fixation. Depending on whether the new, strongly selected mutation occurs on a chromosome carrying an $A_{1}$ or $A_{2}$ allele, the frequency of $A_{1}$ will either increase from $p$ to 1 with probability $p$ or decrease from $p$ to 0 with probability $1-p$. Here we imagine that the selective advantage of the favored mutation is so strong that this change can be treated as a jump. The pseudohitchhiking model 
introduced by Gillespie [10] belongs to this class, as does a related, more general model studied by Kim [15].

The second scenario concerns demographic bottlenecks that occur during transmission of parasites from infected to uninfected hosts. Here we will let $p$ denote the frequency of $A_{1}$ in a chronological series of infected hosts linked by a transmission chain, and we will assume that $p(\cdot)$ can be modeled by a diffusion process from the time when one of these hosts is first infected to the time when that host first transmits the infection to the next host in the transmission chain. Suppose that transmissions occur at rate $\lambda$, and that each new infection is founded by a single parasite, as has been proposed for HIV-1 [31] and for some bacterial pathogens [29]. In this case, $p$ will jump to 0 or 1 following each transmission depending on the type of transmitted parasite. Also, to allow for the possibility that transmission itself might be selective (see, e.g. [27]), we will let $w(p)$ denote the probability that the transmitted parasite is of type $A_{1}$ given that the frequency of this allele in the transmitting host is $p$. In general, we stipulate that $w(0)=0, w(1)=1$, and that $w(p)$ is monotonically increasing. If transmission is unbiased then $w(p)=p$, as in the pseudohitchhiking model. A particular case of this transmission chain model was studied by Rouzine and Coffin [28] to understand the effects of selection and transmission bottlenecks on antigenic variation in HIV-1.

Both of these scenarios can be modeled by a jump diffusion process with infinitesimal generator

$$
\begin{aligned}
G \phi(p)= & \frac{1}{2} p(1-p) \phi^{\prime \prime}(p)+\left(\mu_{0}(1-p)-\mu_{1} p+s(p) p(1-p)\right) \phi^{\prime}(p) \\
& +\lambda w(p)(\phi(1)-\phi(p))+\lambda(1-w(p))(\phi(0)-\phi(p)),
\end{aligned}
$$

where, for technical reasons, we will assume that $s(p)$ and $w(p)$ are smooth functions on $[0,1]$, and that both mutation rates, $\mu_{0}$ and $\mu_{1}$, are positive. Under these conditions, it can be shown (cf. Lemma 3.1) that the process $p(\cdot)$ has a unique stationary distribution, $\pi(p) \mathrm{d} p$, which has a density on $[0,1]$. To characterize the structured coalescent process corresponding to this model, we need to identify the stationary time reversal of the process $p(\cdot)$. Formally, this can be done by solving the following adjoint problem for the operator $\tilde{G}$ :

$$
\int_{0}^{1} \psi(p) G \phi(p) \pi(p) \mathrm{d} p=\int_{0}^{1} \phi(p) \tilde{G} \psi(p) \pi(p) \mathrm{d} p,
$$

where $\phi$ is in the domain of $G$. If $\tilde{G}$ generates a Markov process $\tilde{p}(\cdot)$ then this process will have the same law as the stationary time reversal of $p(\cdot)[23]$. When $\lambda=0, p(\cdot)$ is a diffusion process and a simple calculation using integration by parts shows that $\tilde{G}=G$, demonstrating that the law of the diffusion is invariant under time reversal, as remarked above. However, if $\lambda>0$ then, for the adjoint condition (1.3) to be satisfied for all $\phi \in \mathcal{C}^{2}(\mathbb{R}) \cap \mathcal{C}[0,1]$, we must instead set

$$
\tilde{G} \psi(p)=\frac{1}{2} p(1-p) \psi^{\prime \prime}(p)+\tilde{\mu}(p) \psi^{\prime}(p),
$$

where

$$
\tilde{\mu}(p)=\frac{1}{\pi(p)}\left(p(1-p) \pi^{\prime}(p)+(1-2 p-\mu(p)) \pi(p)\right),
$$

and $\psi \in \mathcal{C}^{2}(\mathbb{R}) \cap \mathcal{C}[0,1]$ satisfies

$$
\psi(1)=\int_{0}^{1} \psi(p) \frac{w(p) \pi(p)}{\kappa} \mathrm{d} p \quad \text { and } \quad \psi(0)=\int_{0}^{1} \psi(p) \frac{(1-w(p)) \pi(p)}{1-\kappa} \mathrm{d} p
$$


with $\kappa=\int_{0}^{1} w(p) \pi(p) \mathrm{d} p$. Although it is not immediately clear that the operator defined by (1.4) is the generator of a Markov process, this calculation does show that the process incorporating bottlenecks is not invariant under time reversal.

To gain some insight into the qualitative behavior of the time-reversed process, it is useful to consider two heuristic descriptions. We begin by observing that the behavior of $\tilde{p}(\cdot)$ depends strongly on whether the boundary points $\{0,1\}$ are accessible or inaccessible to the diffusive motion of the forward process. Recall that, for the Wright-Fisher diffusion corresponding to $A$ (which we call the diffusive motion of the jump diffusion process), Feller's boundary classification conditions show that 0 and 1 are accessible if and only if $u_{0}<\frac{1}{2}$ and $u_{1}<\frac{1}{2}$, respectively; see, e.g. Section 4.7 of [8]. The importance of this distinction is illustrated in Figure 1, which shows sample paths of the jump diffusion process corresponding to cases where the two boundaries are either (a) inaccessible or (b) accessible to the forward diffusion.
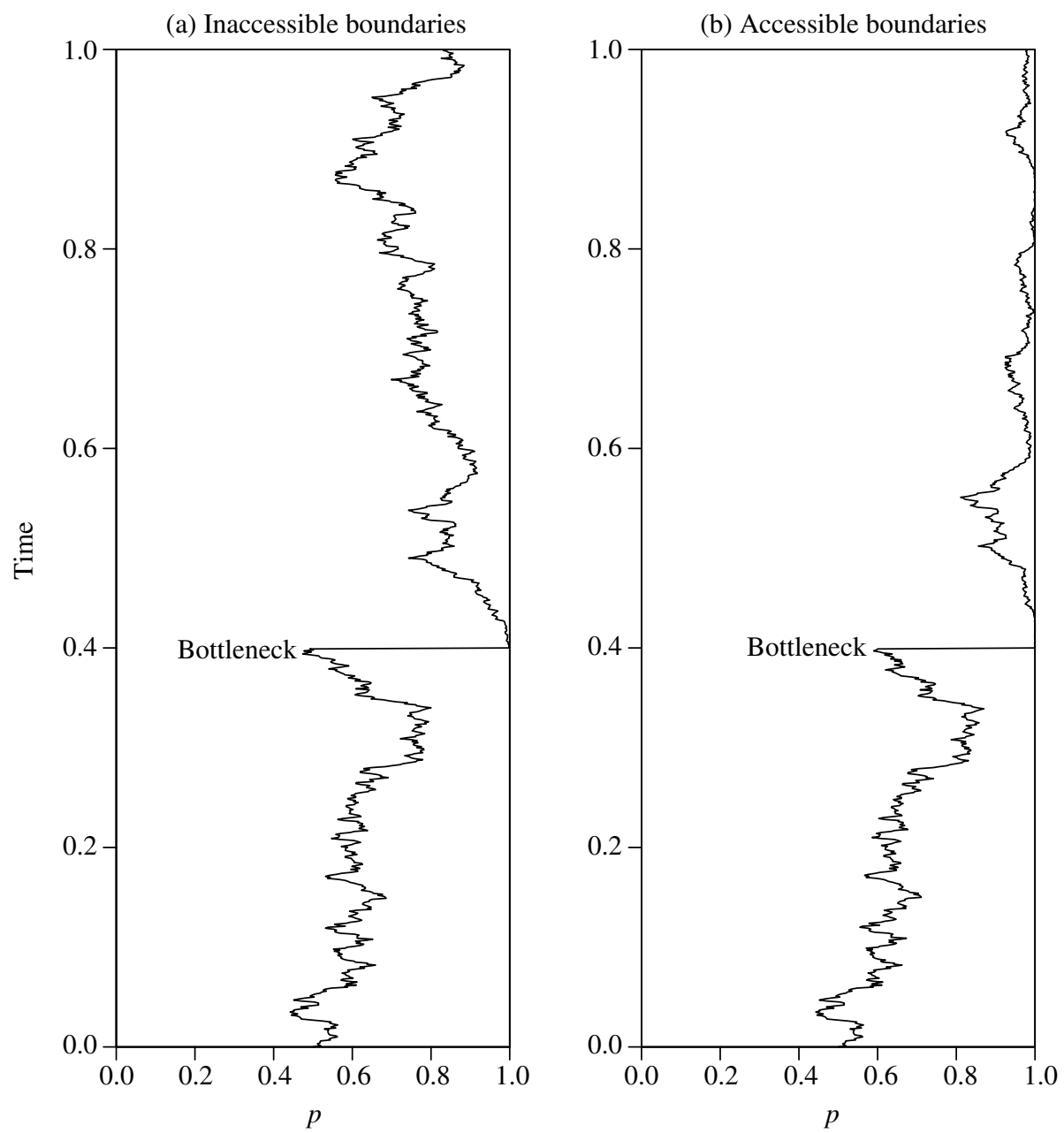

FIGURE 1: Sample paths of the jump diffusion process (1.2) with either (a) inaccessible or (b) accessible boundaries. The forward diffusion is a neutral Wright-Fisher process with symmetric mutation: $\mu_{0}=$ $\mu_{1}=1$ in (a) and 0.2 in (b). 
To see what this suggests about the behavior of the time-reversed process, begin at the top of each figure and follow the sample path backwards in time towards the bottom. If the boundaries are inaccessible then whenever the sample path is followed back to a boundary at some time, the forward process will necessarily have reached that boundary via a jump. Consequently, the time-reversed process must immediately jump into the interval $(0,1)$ whenever it arrives at a boundary that is inaccessible to the forward diffusion. The behavior of the time-reversed process at a boundary that is accessible to the forward diffusion is very different. In this case, when the sample path of the time-reversed jump diffusion hits that boundary, the forward process may have arrived there either diffusively or via a jump from the interior (Figure 1(b)). Accordingly, the time-reversed process need not immediately jump into the interior $(0,1)$ when it visits the boundary, although jumps can only occur when the process is on the boundary and are certain to occur at some such times if $\lambda>0$.

A more quantitative picture of this second case can be obtained by considering a less singular process that approximates the jump diffusion process corresponding to (1.2). For $\epsilon \in\left(0, \frac{1}{2}\right)$, let $p_{\epsilon}(\cdot)=\left(p_{\epsilon}(t): t \geq 0\right)$ be a perturbation of a Wright-Fisher diffusion which at rate $\lambda$ jumps to a point chosen uniformly at random from an interval of width $\epsilon$ adjacent to one of the two boundaries. More precisely, let $p_{\epsilon}(\cdot)$ be the Markov process with generator

$$
\begin{aligned}
G_{\epsilon} \phi(p)= & \frac{1}{2} p(1-p) \phi^{\prime \prime}(p)+\left(\mu_{0}(1-p)-\mu_{1} p+s(p) p(1-p)\right) \phi^{\prime}(p) \\
& +\lambda\left(w(p) \frac{1}{\epsilon} \int_{1-\epsilon}^{1}(\phi(q)-\phi(p)) \mathrm{d} q+(1-w(p)) \frac{1}{\epsilon} \int_{0}^{\epsilon}(\phi(q)-\phi(p)) \mathrm{d} q\right) .
\end{aligned}
$$

Writing $\pi_{\epsilon}(p)$ for the density of the stationary distribution of this process, a simple calculation using (1.3) shows that the stationary time reversal of $p_{\epsilon}(\cdot)$, denoted $\tilde{p}_{\epsilon}(\cdot)$, is also a jump diffusion process with generator

$$
\begin{aligned}
\tilde{G}_{\epsilon} \psi(p)= & \frac{1}{2} p(1-p) \psi^{\prime \prime}(p)+\frac{1}{\pi_{\epsilon}(p)}\left(p(1-p) \pi_{\epsilon}^{\prime}(p)+(1-2 p-\mu(p)) \pi_{\epsilon}(p)\right) \psi^{\prime}(p) \\
& +\lambda \kappa_{\epsilon} \frac{1}{\epsilon \pi_{\epsilon}(p)} \mathbf{1}_{(1-\epsilon, 1]}(p) \int_{0}^{1} \frac{w(q) \pi_{\epsilon}(q)}{\kappa_{\epsilon}}(\psi(q)-\psi(p)) \mathrm{d} q \\
& +\lambda\left(1-\kappa_{\epsilon}\right) \frac{1}{\epsilon \pi_{\epsilon}(p)} \mathbf{1}_{[0, \epsilon)}(p) \int_{0}^{1} \frac{(1-w(q)) \pi_{\epsilon}(q)}{1-\kappa_{\epsilon}}(\psi(q)-\psi(p)) \mathrm{d} q,
\end{aligned}
$$

where $\psi \in \mathcal{C}^{2}([0,1])$ and $\kappa_{\epsilon}=\int_{0}^{1} w(p) \pi_{\epsilon}(p) \mathrm{d} p$. It is easy to read off the behavior of this process from its generator. In particular, we see that $\tilde{p}_{\epsilon}(\cdot)$ can only jump when it is present in the region $[0, \epsilon) \cup(1-\epsilon, 1]$ and that the rate at which jumps occur out of this region is equal to $\lambda \kappa_{\epsilon} /\left(\epsilon \pi_{\epsilon}(p)\right)$ when $p \in(1-\epsilon, 1]$ and $\lambda\left(1-\kappa_{\epsilon}\right) /\left(\epsilon \pi_{\epsilon}(p)\right)$ when $p \in[0, \epsilon)$.

To relate these observations to the process $\tilde{p}(\cdot)$, let $T>0$ and note that, as $\epsilon$ tends to 0 , the sequence of processes $\left(p_{\epsilon}(\cdot)\right)$ converges in distribution on $D_{[0,1]}([0, T])$ to $p(\cdot)$. Furthermore, because time reversal is a continuous mapping on $D_{[0,1]}([0, T])$, the continuous mapping theorem [7] implies that the sequence of processes $\left(\tilde{p}_{\epsilon}(\cdot)\right)$ converges in distribution to the process $\tilde{p}(\cdot)$. In particular, this suggests that $\tilde{p}(\cdot)$ has the following behavior. For each $\epsilon \in\left(0, \frac{1}{2}\right)$, define the additive functionals

$$
\begin{aligned}
& L_{1, \epsilon}(t) \equiv \frac{1}{\epsilon} \int_{0}^{t} \frac{1}{\pi(\tilde{p}(s))} \mathbf{1}_{(1-\epsilon, 1]}(\tilde{p}(s)) \mathrm{d} s, \\
& L_{0, \epsilon}(t) \equiv \frac{1}{\epsilon} \int_{0}^{t} \frac{1}{\pi(\tilde{p}(s))} \mathbf{1}_{[0, \epsilon)}(\tilde{p}(s)) \mathrm{d} s,
\end{aligned}
$$


and suppose that, for $i=0,1$, the limits $L_{i}(t)=\lim _{\epsilon \rightarrow 0} L_{i, \epsilon}(t)$ exist for all $t \geq 0$. Here we would like to interpret $L_{i}(t)$ as the local time of the process $\tilde{p}(\cdot)$ at $i \in\{0,1\}$. Then, by comparison with the jump diffusion processes $\tilde{p}_{\epsilon}(\cdot)$, we expect that if both boundaries are accessible then $\tilde{p}(\cdot)$ is a jump diffusion with diffusive motion in $(0,1)$ governed by $(1.4)$, which jumps from the boundary point 0 to a random point in the interval $(0,1)$ distributed as $w(q) \pi(q) \mathrm{d} q / \kappa$ as soon as $L_{0}(\cdot)$ exceeds an exponential random variable with parameter $\lambda \kappa$, and which jumps from the boundary point 1 to a random point distributed as $(1-w(q)) \pi(q) \mathrm{d} q /(1-\kappa)$ on $(0,1)$ as soon as $L_{1}(\cdot)$ exceeds an exponential random variable with parameter $\lambda(1-\kappa)$. Although these remarks are purely heuristic, we show below that they correctly describe the stationary time reversal of the pseudohitchhiking model and other jump diffusions with generators of the form (1.2).

\section{Main result}

Although our principle concern is with the modified Wright-Fisher process corresponding to (1.2), we state our results for a more general class of jump diffusion processes, which we now introduce. Let the forward process $(p(t): t \geq 0)$ be the jump diffusion process on $[0,1]$ corresponding to the generator

$$
G \phi(p)=\frac{1}{2} v(p) \phi^{\prime \prime}(p)+\mu(p) \phi^{\prime}(p)+\lambda w_{0}(p)(\phi(0)-\phi(p))+\lambda w_{1}(p)(\phi(1)-\phi(p))
$$

for $\phi \in \mathcal{C}^{2}([0,1])$. In other words, the diffusive motion of $p(\cdot)$ is governed by the generator

$$
A \phi(p)=\frac{1}{2} v(p) \phi^{\prime \prime}(p)+\mu(p) \phi^{\prime}(p), \quad \phi \in \mathcal{C}^{2}([0,1])
$$

with infinitesimal drift and variance coefficients, $\mu(\cdot)$ and $v(\cdot)$, respectively, while jumps occur at a constant rate $\lambda \geq 0$ and move the process from state $p \in[0,1]$ either to 0 with probability $w_{0}(p) \in[0,1]$ or to 1 with probability $w_{1}(p):=1-w_{0}(p)$. Throughout this paper, we will make the following assumption.

Assumption 2.1. The infinitesimal mean and variance satisfy $\mu(0)>0>\mu(1)$ and $v(0)=$ $v(1)=0<v(p)$ for all $p \in(0,1)$, respectively. Furthermore, $v(\cdot), \mu(\cdot)$, and $w_{0}(\cdot)$ are analytic functions in a neighborhood of [0,1], and the infinitesimal variance has nonzero derivatives, $v^{\prime}(0)>0>v^{\prime}(1)$, at the boundaries.

For example, if $A$ is the generator of a neutral Wright-Fisher diffusion (1.1) (with $s(p) \equiv 0)$, then Assumption 2.1 is satisfied with $\mu(0)=\mu_{0}>0, \mu(1)=-\mu_{1}<0$, and $v^{\prime}(0)=$ $1=-v^{\prime}(1)$. We also remark that, when Assumption 2.1 is satisfied, Lemma 3.1 shows that $(p(t): t \geq 0)$ has a unique stationary distribution $\pi(p) \mathrm{d} p$ with a density $\pi(\cdot)$ that satisfies a second-order ordinary differential equation with nonlocal boundary conditions.

In Theorem 2.1, we characterize the time-reversed process $(\tilde{p}(t): t \geq 0)$ of the forward process $(p(t): t \geq 0)$. In keeping with the heuristic description given in the introduction, $(\tilde{p}(t): t \geq 0)$ is also a jump diffusion process on $[0,1]$ but now with jumps from the boundary $\{0,1\}$ to the interior $(0,1)$. The diffusive motion of this process is governed by the generator

$$
\tilde{A} \psi(p)=\frac{1}{2} v(p) \psi^{\prime \prime}(p)+\tilde{\mu}(p) \psi^{\prime}(p), \quad \text { where } \quad \tilde{\mu}(p):=-\mu(p)+\frac{(v \pi)^{\prime}(p)}{\pi(p)}
$$

and $\psi \in \mathcal{C}^{2}([0,1])$. Note that this diffusion has the same infinitesimal variance as the forward diffusion, but has a different infinitesimal drift that depends on the jump events via the stationary 
density $\pi(\cdot)$. Also, the jump rates of the time-reversed process depend on a local time process which is described in the following way. Recall that the scale function and the speed measure associated with $\tilde{A}$ are

$$
\tilde{S}(p):=\int_{1 / 2}^{p} \exp \left(-\int_{1 / 2}^{x} \frac{2 \tilde{\mu}(z)}{v(z)} \mathrm{d} z\right) \mathrm{d} x \quad \text { and } \quad \tilde{m}(\mathrm{~d} p):=\frac{1}{v(p) \tilde{S}^{\prime}(p)} \mathrm{d} p, \quad p \in[0,1],
$$

respectively. The scale function will be identified with the associated measure $\tilde{S}(\mathrm{~d} p):=$ $\tilde{S}^{\prime}(p) \mathrm{d} p$ on $[0,1]$ and the speed measure $\tilde{m}(\mathrm{~d} p)$ will be identified with its density function.

We define the local time process of the jump diffusion $\tilde{p}(\cdot)$ such that it agrees with the localtime process of the diffusive motion until the first jump. More formally, we will introduce a nonnegative process $\left(\tilde{L}_{p}(t): t \geq 0, p \in[0,1]\right)$ which is almost surely (a.s.) continuous in $(t, p)$ and which satisfies

$$
\int_{0}^{t} f(\tilde{p}(u)) \mathrm{d} u=\int_{0}^{1} f(p) \tilde{L}_{p}(t) \tilde{m}(\mathrm{~d} p) \quad \text { a.s. for } t \geq 0,
$$

for all measurable $f:[0,1] \rightarrow[0, \infty)$. We remark that the local-time process satisfying $(2.4)$ differs from the semimartingale local time of the diffusive motion of the time-reversed process by a scalar factor (see (7.4) below), i.e. $\tilde{L}$ is a weighted semimartingale local time. That this process is well defined is shown below in Lemma 6.1. The last ingredient needed in our construction is a pair of independent, exponentially distributed random variables, $R_{0}$ and $R_{1}$, with parameters

$$
r_{i}:=\lim _{p \rightarrow i} \frac{\tilde{m}(p)}{\pi(p)} \lambda \kappa_{i} \in[0, \infty],
$$

where $\kappa_{i}:=\int_{0}^{1} w_{i}(p) \pi(p) \mathrm{d} p, i \in\{0,1\}$. The existence of the limit displayed in (2.5) is guaranteed by Lemma 4.3. By convention, $R_{i}:=0$ if $r_{i}=\infty$ and $R_{i}:=\infty$ if $r_{i}=0$.

With these definitions we now describe the dynamics of the time-reversed process $(\tilde{p}(t): t \geq 0)$. Between jump times, $(\tilde{p}(t): t \geq 0)$ evolves according to the law of the diffusion governed by $\tilde{A}$. If this diffusion hits a boundary $i \in\{0,1\}$ at a time $t \geq 0$ and if at that the local-time process exceeds the random variable $R_{i}$, that is, if $\tilde{L}_{i}(t) \geq R_{i}$, then $\tilde{p}(\cdot)$ jumps from $i$ to a random point chosen from $(0,1)$ according to the distribution $\left(1 / \kappa_{i}\right) \int w_{i}(p) \pi(p) \mathrm{d} p$. From this point, $\tilde{p}(\cdot)$ restarts independently of the sample path up to that time.

To better understand how the dynamics of $\tilde{p}(\cdot)$ are influenced by the boundary behavior of the forward process, we take a closer look at the jump times. Because the coefficients $v(\cdot)$ and $\mu(\cdot)$ are smooth on an interval containing $[0,1]$, an application of Feller's boundary classification criteria shows that a boundary point $i \in\{0,1\}$ is accessible to the forward diffusive motion if and only if $2|\mu(i)|<\left|v^{\prime}(i)\right|$. Then, in conjunction with Lemma 3.2 below, which describes the asymptotics of the density $\pi(p)$ near the boundaries, Lemma 4.3 below implies that

$$
r_{i}:=\lim _{p \rightarrow i} \frac{\tilde{m}(p)}{\pi(p)} \lambda \kappa_{i} \begin{cases}\in(0, \infty) & \text { if } 2|\mu(i)|<\left|v^{\prime}(i)\right| \text { and } \lambda w_{i}(\cdot) \not \equiv 0 \\ =\infty & \text { if } 2|\mu(i)| \geq\left|v^{\prime}(i)\right| \text { and } \lambda w_{i}(\cdot) \not \equiv 0 \\ =0 & \text { if } \lambda w_{i}(\cdot) \equiv 0\end{cases}
$$

for $i \in\{0,1\}$. Thus, provided that $\lambda w_{i}(\cdot) \not \equiv 0$, the time-reversed process immediately jumps into the interior $(0,1)$ if the boundary point is inaccessible to the forward diffusive motion, that is, if $2|\mu(i)| \geq\left|v^{\prime}(i)\right|$. In this case, the state space of $\tilde{p}(\cdot)$ is in fact $[0,1] \backslash\{i\}$. In contrast, if $i$ is accessible to the forward diffusion and $\lambda w_{i}(\cdot)>0$, then the exponential random variable 
$R_{i}$ is a.s. positive, and so a positive amount of local time will have to be accrued at $i$ before a jump occurs off of this boundary point.

Note that, in either case, we expect that both boundary points are accessible to the backward diffusive motion. According to Lemma 4.1 below,

$$
\tilde{\mu}(i)=\left\{\begin{array}{ll}
\mu(i) & \text { if } 2|\mu(i)| \leq\left|v^{\prime}(i)\right|, \\
v^{\prime}(i)-\mu(i) & \text { if } 2|\mu(i)| \geq\left|v^{\prime}(i)\right|,
\end{array} \quad i \in\{0,1\}\right.
$$

and, again, an application of Feller's boundary criteria shows that the boundary point $i$ is accessible to the backward diffusive motion whenever $2 \mu(i) \neq v^{\prime}(i)$. The critical case is more subtle. Then, $2 \tilde{\mu}(i)=v^{\prime}(i)$, and so $i$ would be inaccessible if the drift coefficient $\tilde{\mu}(\cdot)$ were analytic in a neighborhood of $i$. However, we show in Lemma 4.1 that

$$
\tilde{\mu}(p)=\mu(i)+\frac{v^{\prime}(i)}{\ln (|p-i|)}+O\left(\frac{|p-i|}{\ln |p-i|}\right),
$$

and then Feller's criteria reveal that the logarithmic singularity is just sufficient to render the point $i$ accessible to the backward diffusive motion when $2 \tilde{\mu}(i)=v^{\prime}(i)$.

Our main result states that the process $\tilde{p}(\cdot)$ has the same law as the stationary time reversal of the jump diffusion $p(\cdot)$.

Theorem 2.1. Suppose that Assumption 2.1 holds. Let $p(\cdot)$ be the jump diffusion on $[0,1]$ with generator $G$ as defined in (2.1). Then the process $(\tilde{p}(t): t \geq 0)$ is a version of the stationary time reversal of $(p(t): t \geq 0)$, that is,

$$
(\tilde{p}(t): t \leq T) \stackrel{\mathrm{D}}{=}(p(T-t): t \leq T) \text { for all } T \geq 0
$$

if the distribution of $p(0)$ is the stationary distribution $\pi(p) \mathrm{d} p$. Here ' $=$ ' denotes equality in distribution.

The proof of Theorem 2.1 is deferred to Section 7 .

Theorem 2.1 establishes the time reversal of the stationary process over a fixed time interval $[0, T], T<\infty$, fixed and nonrandom. Readers interested in other pathwise time reversals are referred to the literature. It has been shown that processes which are in 'Hunt duality' (see [4, Chapter VI]) are time reversals of each other. Reversing time at the end point of an excursion from an accessible boundary point results in the dual process being started at this boundary point; see [9] and [21]. The paper by Mitro [22] reverses time at inverse local time points.

The remainder of the paper is organized as follows. The next section collects some results concerning the stationary distribution of the jump diffusion process (1.2). In Section 4 we describe the boundary behavior of $\tilde{p}(\cdot)$. In particular, we show that the time-reversed process jumps immediately off of any boundary that is inaccessible to the forward diffusion. In Section 5 we identify a core for the generator $\tilde{G}$ satisfying the adjoint condition (1.3). The local-time process of $\tilde{p}(\cdot)$ is introduced and studied in Section 6. Finally, in Section 7 we show that $\tilde{p}(\cdot)$ has generator $\tilde{G}$. The proof of this result depends on an application of the Itô-Tanaka formula.

\section{The stationary distribution}

The following lemma asserts that, if the conditions of Assumption 2.1 are satisfied then the jump diffusion process $p(\cdot)$ has a unique stationary distribution on $[0,1]$. It is also shown that 
this distribution has a density $\pi(\cdot)$ with respect to Lebesgue measure which satisfies a secondorder ordinary differential equation (ODE) subject to boundary conditions that are nonlocal whenever $\lambda>0$. If $\lambda=0$ then this equation can be solved explicitly, leading to the familiar expression

$$
\pi(p)=C^{-1} \frac{1}{v(p)} \exp \left(2 \int^{p} \frac{\mu(q)}{v(q)} \mathrm{d} q\right),
$$

where $C$ is a normalizing constant; see, e.g. Section 4.5 of [8]. Although a general closed-form expression for $\pi(\cdot)$ apparently does not exist when $\lambda>0, \pi(\cdot)$ can be calculated by numerically solving (3.1a)-(3.1e), below, using a modification of the shooting method [25]. In addition, below we give an explicit formula for the stationary density in the important special case of a neutral Wright-Fisher diffusion subject to recurrent bottlenecks.

Lemma 3.1. Suppose that Assumption 2.1 holds. Then there exists a unique stationary distribution for the process $(p(t): t \geq 0)$. This distribution is given by $\mathbf{1}_{(0,1)}(p) \pi(p) \mathrm{d} p$, where $\pi:(0,1) \rightarrow(0, \infty)$ is the unique solution of the nonlocal boundary value problem

$$
\begin{gathered}
\left(\left(\frac{1}{2} v \pi\right)^{\prime \prime}-(\mu \pi)^{\prime}-\lambda \pi\right)(p)=0 \text { for all } p \in(0,1), \\
\lim _{p \rightarrow 0}\left(\mu \pi-\left(\frac{1}{2} v \pi\right)^{\prime}\right)(p)=\lambda \kappa_{0}, \\
\lim _{p \rightarrow 1}\left(\mu \pi-\left(\frac{1}{2} v \pi\right)^{\prime}\right)(p)=-\lambda \kappa_{1}, \\
\lim _{p \rightarrow 0}(v \pi)(p)=0=\lim _{p \rightarrow 1}(v \pi)(p) \\
\int_{0}^{1} \pi(p) \mathrm{d} p=1
\end{gathered}
$$

where $\kappa_{i}:=\int_{0}^{1} w_{i}(p) \pi(p) \mathrm{d} p$ for $i \in\{0,1\}$. Furthermore, $p(t)$ converges in distribution to the stationary distribution as $t \rightarrow \infty$ for every initial distribution of $p(0)$.

Proof. Existence and uniqueness of a stationary distribution $\bar{\pi}(\mathrm{d} p)$ follow from standard arguments, so we give only a sketch. Couple two versions of $(p(t): t \geq 0)$ with different initial distributions through the same jump times such that the diffusive motions inbetween jumps are independent until they first meet and are identical thereafter. Owing to the assumption that $\mu(0)>0>\mu(1)$, the coupling is successful if there are no jumps, that is, if $\lambda=0$; see Theorem V.54.5 of [26]. In the presence of jumps $(\lambda>0)$, the probability that both components jump to the same boundary is positive at every jump and, therefore, the two components agree eventually. As a consequence of this successful coupling and of the compactness of $[0,1], p(t)$ converges in distribution to a probability measure $\bar{\pi}(\mathrm{d} p)$ as $t \rightarrow \infty$ and $\bar{\pi}(\mathrm{d} p)$ is an invariant distribution.

Next we prove that $\bar{\pi}(\cdot)$ has a smooth density $\pi(\cdot)$. Denote by $(X(t))_{t \geq 0}$ the diffusion governed by $A$ (see (1.1)). The scale function and the speed measure associated with $A$ are

$$
S(p):=\int_{1 / 2}^{p} \exp \left(-\int_{1 / 2}^{x} \frac{2 \mu(z)}{v(z)} \mathrm{d} z\right) \mathrm{d} x \quad \text { and } \quad m(p) \mathrm{d} p:=\frac{1}{v(p) S^{\prime}(p)} \mathrm{d} p, \quad p \in[0,1]
$$

respectively. Existence and smoothness of the density $\pi(\cdot)$ will be derived from existence and uniqueness of the transition density $Q(t ; p, q)$ of $(X(t))_{t \geq 0}$ with respect to the speed measure. Existence of $Q(t ; p, q)$ is established in [11] ([20] is more detailed in a special case) via an eigen-differential expansion. To state this result more formally, we introduce the 
following notation. The interval defined in [11] — here denoted by $I^{\bullet}$ —is the unit interval closed at 0 if 0 is accessible, closed at 1 if 1 is accessible, and open otherwise. For this, note that whenever $(X(t))_{t \geq 0}$ hits a boundary point, it immediately returns to the interior $(0,1)$ because of the assumption that $\mu(0)>0>\mu(1)$. Moreover, note that the stopping time $\min \left\{t \geq 0: X(t) \notin I^{\bullet}\right\}=\infty$ a.s. The generator of $(X(t))_{t \geq 0}$ is defined in [11] via right derivatives. As $(X(t))_{t \geq 0}$ is a regular diffusion, this generator coincides with

$$
A f(p)=\frac{1}{m(p)} \frac{\mathrm{d}}{\mathrm{d} p}\left(\frac{1}{S^{\prime}(p)} f^{\prime}(p)\right), \quad p \in I^{\bullet},
$$

for $f \in \mathcal{C}^{2}\left(I^{\bullet}\right)$. There exists a solution $\mathfrak{e}(\gamma, \cdot)=\left(\mathfrak{e}_{1}(\gamma, \cdot), \mathfrak{e}_{2}(\gamma, \cdot)\right)$ of

$$
\begin{gathered}
(A \mathfrak{e} \gamma, \cdot)(p)=\gamma \mathfrak{e}(\gamma, p) \quad \text { for all } 0<p<1, \\
\mathfrak{e}\left(\gamma, \frac{1}{2}\right)=(1,0), \quad \frac{1}{m(1 / 2)} \mathfrak{e}^{\prime}\left(\gamma, \frac{1}{2}\right)=(0,1),
\end{gathered}
$$

for every $\gamma \in(-\infty, 0]$ such that $\gamma \mapsto \mathfrak{e}(\gamma, p)$ is continuous for every $p \in I^{\bullet}$. Based on these eigenfunctions, it was shown in [11] that there exists a Borel measure $\mathfrak{s}(\mathrm{d} \gamma)$ from $(-\infty, 0]$ to $2 \times 2$ symmetric nonnegative definite matrices

$$
\mathfrak{s}(\mathrm{d} \gamma)=\left(\begin{array}{ll}
\mathfrak{s}_{11}(\mathrm{~d} \gamma) & \mathfrak{s}_{12}(\mathrm{~d} \gamma) \\
\mathfrak{s}_{21}(\mathrm{~d} \gamma) & \mathfrak{s}_{22}(\mathrm{~d} \gamma)
\end{array}\right)
$$

such that

$$
Q(t ; p, q)=\int_{-\infty}^{0} \mathrm{e}^{\gamma t} \mathfrak{e}^{\top}(\gamma, p) \mathfrak{s}(\mathrm{d} \gamma) \mathfrak{e}(\gamma, p), \quad(t, p, q) \in(0, \infty) \times I^{\bullet} \times I^{\bullet},
$$

is the transition density of $(X(t))_{t \geq 0}$ with respect to the speed measure $m(\cdot)$. Now, as our jump diffusion $p(\cdot)$ could also jump to an inaccessible boundary, we need to extend $p \mapsto Q(t ; p, q)$ onto $[0,1]$. Note that if $i \in\{0,1\}$ is inaccessible then $i$ is an entrance boundary due to the assumption that $(-1)^{i} \mu(i)>0$. As in Problem 3.6.3 of [11], we use the Markov property to extend $(X(t))_{t \geq 0}$ to the state space $[0,1]$. Thus, we may assume $Q(t ; p, q)$ to be defined on $(0, \infty) \times[0,1] \times I^{\bullet}$.

With these results on the transition density of $(X(t))_{t \geq 0}$ we now establish the existence of a smooth density of $\bar{\pi}(\cdot)$. Define $\kappa_{1}:=1-\kappa_{0}, \kappa_{0} \in[0,1]$, by

$$
\kappa_{i}:=\int_{0}^{1} w_{i}(p) \bar{\pi}(\mathrm{d} p) \quad \text { for } i \in\{0,1\},
$$

and observe that $\kappa_{i}$ is the probability that a stationary version of the process jumps to the boundary point $i$ when it jumps. Recall that the jump times of $p(\cdot)$ form a Poisson process with rate $\lambda$ and that inbetween jumps, $p(\cdot)$ evolves according to $A$. If $U$ is any Borel measurable set in $[0,1]$ then, by conditioning on the time and distribution of the last jump, we have

$$
\bar{\pi}(U)=\kappa_{0} \int_{0}^{\infty} \lambda \mathrm{e}^{-\lambda t} \int_{U} Q(t ; 0, q) m(q) \mathrm{d} q \mathrm{~d} t+\kappa_{1} \int_{0}^{\infty} \lambda \mathrm{e}^{-\lambda t} \int_{U} Q(t ; 1, q) m(q) \mathrm{d} q \mathrm{~d} t .
$$


Interchanging integrals, we infer that $\bar{\pi}(\cdot)$ has a density with respect to the Lebesgue measure and we set $\pi(q) \mathrm{d} q:=\bar{\pi}(\mathrm{d} q)$, where $\pi:(0,1) \rightarrow[0, \infty)$ satisfies

$$
\begin{aligned}
\pi(q) & =\sum_{j=0}^{1} \kappa_{j} m(q) \int_{0}^{\infty} \lambda \mathrm{e}^{-\lambda t} \int_{-\infty}^{0} \mathrm{e}^{\gamma t} \mathfrak{e}^{\top}(\gamma, j) \mathfrak{s}(\mathrm{d} \gamma) \mathfrak{e}(\gamma, q) \mathrm{d} t \\
& =\sum_{j=0}^{1} \kappa_{j} m(q) \int_{-\infty}^{0} \frac{\lambda}{\lambda-\gamma} \mathfrak{e}^{\top}(\gamma, j) \mathfrak{s}(\mathrm{d} \gamma) \mathfrak{e}(\gamma, q) \mathrm{d} t \\
& =\lambda m(q)\left(\kappa_{0} G_{\lambda}(0, q)+\kappa_{1} G_{\lambda}(1, q)\right) .
\end{aligned}
$$

The function $G_{\lambda}(p, q)$ is Green's function and it is $\mathcal{C}^{2}$ in the second variable for every $p \in[0,1]$. As the speed density $m(\cdot)$ is also $\mathcal{C}^{2}$ in $(0,1)$ due to Assumption 2.1 , we conclude that the stationary density $\pi(\cdot)$ is twice continuously differentiable.

The main step of the proof is to show that $\pi(\cdot)$ satisfies (3.1a)-(3.1d). By Proposition 4.9.2 of [7], the stationary distribution $\pi(p) \mathrm{d} p$ satisfies

$$
\int_{0}^{1} G \phi(p) \pi(p) \mathrm{d} p=0
$$

for all $\phi \in \mathcal{C}^{2}([0,1])$. Let $0<\varepsilon<\frac{1}{2}$. The functions $v, \mu, \phi$, and $\pi$ are $\mathcal{C}^{2}$ in $[\varepsilon, 1-\varepsilon]$. Integration by parts yields

$$
\begin{aligned}
\int_{\varepsilon}^{1-\varepsilon} & G \phi \pi \mathrm{d} p-\phi(0) \int_{\varepsilon}^{1-\varepsilon} \lambda w_{0} \pi \mathrm{d} p-\phi(1) \int_{\varepsilon}^{1-\varepsilon} \lambda w_{1} \pi \mathrm{d} p \\
& =\int_{\varepsilon}^{1-\varepsilon}\left[\phi^{\prime \prime} \cdot\left(\frac{1}{2} v \pi\right)+\phi^{\prime} \cdot(\mu \pi)-\lambda \phi \pi\right] \mathrm{d} p \\
& =\left[\phi^{\prime} \frac{1}{2} v \pi\right]_{\varepsilon}^{1-\varepsilon}+\left[\phi\left(\mu \pi-\left(\frac{1}{2} v \pi\right)^{\prime}\right)\right]_{\varepsilon}^{1-\varepsilon}-\int_{\varepsilon}^{1-\varepsilon} \phi\left[(\mu \pi)^{\prime}-\left(\frac{1}{2} v \pi\right)^{\prime \prime}+\lambda \pi\right] \mathrm{d} p .
\end{aligned}
$$

By considering all functions $\phi \in \mathcal{C}^{2}$ with support in $(\varepsilon, 1-\varepsilon)$ and then letting $\varepsilon \rightarrow 0$, we conclude that $\pi(\cdot)$ satisfies the second-order ODE (3.1a). Furthermore, because the functions $G \phi, w_{0}, w_{1}$ are bounded and $\pi$ is integrable, we may apply the dominated convergence theorem to the integrals on the left-hand side of (3.3) as $\varepsilon \rightarrow 0$. Together with (3.2), this shows that

$$
\begin{gathered}
\lim _{\varepsilon \rightarrow 0}\left[\phi^{\prime} \frac{1}{2} v \pi\right]_{\varepsilon}^{1-\varepsilon}+\phi(1) \lim _{\varepsilon \rightarrow 0}\left(\mu \pi-\left(\frac{1}{2} v \pi\right)^{\prime}\right)(1-\varepsilon)-\phi(0) \lim _{\varepsilon \rightarrow 0}\left(\mu \pi-\left(\frac{1}{2} v \pi\right)^{\prime}\right)(\varepsilon) \\
=-\phi(1) \lambda \kappa_{1}-\phi(0) \lambda \kappa_{0} .
\end{gathered}
$$

As $\phi$ was arbitrary, this implies the nonlocal boundary conditions (3.1b)-(3.1d).

If $\hat{\pi}(\cdot)$ is another normalized solution of (3.1a)-(3.1e) then reversing the previous arguments shows that (3.2) holds with $\pi$ replaced by $\hat{\pi}(\cdot)$. This in turn implies that $\hat{\pi}(p) \mathrm{d} p$ is another stationary distribution and we conclude that $\hat{\pi}=\pi$. It remains to show that $\pi(\cdot)$ is strictly positive. Assuming that $\pi(p)=0$ for some $p \in(0,1)$, we conclude that $\pi^{\prime}(p)=0$ from $p$ being necessarily a global minimum. However, the only solution of the second-order ODE (3.1a) satisfying $\pi(p)=0=\pi^{\prime}(p)$ is the zero function, which contradicts the assumption that $\pi(\cdot)$ is a probability density. This completes the proof. 
Remark 3.1. Lemma 3.1 can be used to find an explicit formula for $\pi(\cdot)$ when the jump diffusion process is a model of a neutrally evolving population subject to recurrent bottlenecks, i.e. when $p(\cdot)$ has generator

$G \phi(p)=\frac{1}{2} p(1-p) \phi^{\prime \prime}(p)+\left(\mu_{0}(1-p)-\mu_{1} p\right) \phi^{\prime}(p)+\lambda(p \phi(1)+(1-p) \phi(0)-\phi(p))$.

In this case, (3.1a) is a hypergeometric equation and, using the fact that the mean frequency of allele $A_{1}$ in a stationary population is $\mu_{0} /\left(\mu_{0}+\mu_{1}\right)$, we find that the density $\pi(p)$ is equal to $\pi(p)=C^{-1} p^{2 \mu_{0}-1}(1-p)^{2 \mu_{1}-1}\left[\mu_{0} F\left(1-a, 1-b, 2 \mu_{0}, p\right)+\mu_{1} F\left(1-a, 1-b, 2 \mu_{1}, 1-p\right)\right]$,

where $C$ is a normalizing constant, $F(a, b, c ; z)$ is Gauss' hypergeometric function, and the constants $a$ and $b$ are determined (up to interchange) by the equations $a+b=3-2\left(\mu_{0}+\mu_{1}\right)$ and $a b=2\left(\lambda+1-\mu_{0}-\mu_{1}\right)$.

The second lemma of this section provides information on the boundary behavior of the density of the stationary distribution. This information is derived using results on second-order ODEs with regular singular points.

We adopt the Landau big-O and little-o notation. In addition, for two functions $\psi_{1}(\cdot)$ and $\psi_{2}(\cdot)$, we write $\psi_{1}(p) \sim \psi_{2}(p)$ as $p \rightarrow i$ if both $\psi_{1}(p)=O\left(\psi_{2}(p)\right)$ and $\psi_{2}(p)=O\left(\psi_{1}(p)\right)$ as $p \rightarrow i$.

Lemma 3.2. Suppose that Assumption 2.1 holds. Let $\pi(\cdot)$ be the density of the stationary distribution of the jump diffusion $p(\cdot)$ corresponding to generator (2.1). Then, for $i \in\{0,1\}$, $\pi(\cdot)$ is equal to

$$
\pi(p)= \begin{cases}C_{i}|p-i|^{\left(2 \mu(i)-v^{\prime}(i)\right) / v^{\prime}(i)}+O(1) & \text { if } 2|\mu(i)|<\left|v^{\prime}(i)\right|, \\ \frac{2 \lambda \kappa_{i}}{\left|v^{\prime}(i)\right|} \ln \left(\frac{1}{|p-i|}\right)+O(1) & \text { if } 2 \mu(i)=v^{\prime}(i), \\ \frac{2 \lambda \kappa_{i}}{\left|2 \mu(i)-v^{\prime}(i)\right|} & \\ +O\left(|p-i|+|p-i|^{\left(2 \mu(i)-v^{\prime}(i)\right) / v^{\prime}(i)}\right) & \text { if } 2|\mu(i)|>\left|v^{\prime}(i)\right| \text { and } \lambda w_{i} \not \equiv 0, \\ C_{i}|p-i|^{\left(2 \mu(i)-v^{\prime}(i)\right) / v^{\prime}(i)} & \\ +O\left(|p-i|^{2 \mu(i) / v^{\prime}(i)}\right) & \text { if } 2|\mu(i)|>\left|v^{\prime}(i)\right| \text { and } \lambda w_{i} \equiv 0,\end{cases}
$$

as $p \rightarrow i$, where $C_{i} \in(0, \infty)$. In addition, if $2 \mu(i)=v^{\prime}(i)$ and $\lambda w_{i} \equiv 0$, then $\pi(i)>0$.

Proof. We only consider $i=0$ as the case $i=1$ is analogous. We begin by observing that $i=0$ is a regular singular point for the differential equation (3.1a); see, e.g. Section 9.6 of [3] for this concept. The associated indicial equation for 0 is

$$
v(v-1)+2 \frac{v^{\prime}(0)-\mu(0)}{v^{\prime}(0)} v=0,
$$

and has roots $\alpha:=0$ and $\beta:=\left(2 \mu(0)-v^{\prime}(0)\right) / v^{\prime}(0)$. Note that $\beta>-1$. If $(\alpha-\beta) \notin \mathbb{Z}$ then Theorem IX.7 of [3] tells us that $\pi(\cdot)$ is equal to a linear combination of $b_{1}(p):=p^{\alpha}\left(1+h_{1}(p)\right)$ and $b_{2}(p):=p^{\beta}\left(1+h_{2}(p)\right)$ in a neighborhood of 0 , where $h_{1}$ and $h_{2}$ are suitable analytic functions satisfying $h_{1}(0)=0=h_{2}(0)$.

If $(\alpha-\beta) \in \mathbb{Z}$ then Theorem IX.8 of [3] tells us that $\pi(\cdot)$ is equal to a linear combination of $b_{1}(p), b_{2}(p)$, and $\ln (p) b_{1}(p)$ in a neighborhood of 0 . If $2 \mu(0) / v^{\prime}(0) \in \mathbb{N}_{\geq 2}$ then, assuming 
that $\pi(p)=-c_{1} \ln (p)+O(1), \pi^{\prime}(p)=-c_{1} / p+O(1)$ for some constant $c_{1}>0$ leads to the contradiction

$$
\infty>\lambda \kappa_{0}=\left(\mu(0)-\frac{1}{2} v^{\prime}(0)\right) \lim _{p \rightarrow 0} \pi(p)-\frac{1}{2} v^{\prime}(0) \lim _{p \rightarrow 0} p \pi^{\prime}(p)=\infty,
$$

where we have used (3.1b). Therefore, $\ln (p)$ does not contribute to $\pi(\cdot)$ if $2 \mu(0)>v^{\prime}(0)$.

It remains to calculate the coefficients. In the case $2 \mu(0) \neq v^{\prime}(0)$, insert $\pi(b)=c_{1} b_{1}(p)+$ $c_{2} b_{2}(p)$ into (3.1b) to obtain the coefficient $c_{1}$, i.e.

$$
\begin{aligned}
\lambda \kappa_{0} & =\lim _{p \rightarrow 0}\left[\left(\mu(p)-\frac{1}{2} v^{\prime}(p)\right) \pi(p)-\frac{1}{2} v(p) \pi^{\prime}(p)\right] \\
& =\lim _{p \rightarrow 0}\left[\left(\mu(0)-\frac{1}{2} v^{\prime}(0)\right)\left(c_{1}+c_{2} p^{\beta}\right)-\frac{1}{2} v^{\prime}(0) p c_{2} \beta p^{\beta-1}\right] \\
& =\left(\mu(0)-\frac{1}{2} v^{\prime}(0)\right) c_{1} .
\end{aligned}
$$

Of course, if $\lambda \kappa_{0}=0$ then $\pi(\cdot) \not \equiv 0$ implies that $c_{2}>0$. Next we show that $\lambda \kappa_{0}>0$ together with $2 \mu(0)<v^{\prime}(0)$ implies that $c_{2}>0$.

Assuming that $c_{2}=0$ implies that $\pi(0)=2 \lambda \kappa_{0} /\left(2 \mu(0)-v^{\prime}(0)\right)<0$, which contradicts $\pi(\cdot)$ being a density function. In the critical case, $2 \mu(0)=v^{\prime}(0),(3.1 \mathrm{~b})$ implies that

$$
\lambda \kappa_{0}=-\frac{1}{2} v^{\prime}(0) \lim _{p \rightarrow 0}\left(p \pi^{\prime}(p)\right) .
$$

Therefore, the coefficient of $-\ln (p)$ is $2 \lambda \kappa_{0} / v^{\prime}(0)$. If $2 \mu(0)=v^{\prime}(0)$ and $\lambda \kappa_{0}=0$, then assuming that $\pi(0)=0$ implies that $\pi(p)=c p^{n}+O\left(p^{n+1}\right)$ with $c \neq 0$ and $n \geq 1$. Inserting this into the ODE (3.1a) leads to

$$
\begin{aligned}
0 & =\frac{1}{2} v^{\prime}(0) c\left(p^{n+1}\right)^{\prime \prime}-\mu(0) c\left(p^{n}\right)^{\prime}+O\left(p^{n}\right) \\
& =\frac{(n+1) n}{2} v^{\prime}(0) c p^{n-1}-n \frac{v^{\prime}(0)}{2} p^{n-1}+O\left(p^{n}\right)
\end{aligned}
$$

as $p \rightarrow 0$. Dividing by $n v^{\prime}(0) c p^{n-1} / 2$ and letting $p \rightarrow 0$ results in the contradiction $n+1=1$. This completes the proof.

\section{Boundary behavior of the time-reversed process}

We begin this section by characterizing the boundary behavior of the infinitesimal drift coefficient of the time-reversed process. This information is of interest for two reasons. First, it will be used to establish that any boundary point that is accessible to the forwards-in-time process, either diffusively or via jumps, is accessible to the diffusive motion of the time-reversed process. Secondly, we also expect the time-reversed process to have the same state space, [0, 1], as the forward process. Indeed, if a boundary point $i$ is inaccessible to the forward diffusive motion then subsequent results will show that the time-reversed process jumps back into the interior as soon as it hits a boundary. If, however, $i$ is accessible to the forward diffusive motion then, because the time-reversed process may visit $i$ without jumping, we need to confirm that $\tilde{p}(\cdot)$ does not then wander outside of $[0,1]$. To this end, we will show that $\tilde{\mu}(0) \geq 0$ whenever 0 is accessible and, similarly, that $\tilde{\mu}(1) \leq 0$ whenever 1 is accessible. 
Lemma 4.1. Suppose that Assumption 2.1 holds. Then the drift function $\tilde{\mu}(\cdot)$ of the backward diffusive motion defined in (2.2) satisfies

$$
\tilde{\mu}(p)= \begin{cases}\mu(i)+O(|p-i|) & \text { if } 2|\mu(i)|<\left|v^{\prime}(i)\right| \text { or } \lambda \kappa_{i}=0, \\ \mu(i)+\frac{v^{\prime}(i)}{\ln (|p-i|)}+O\left(\frac{|p-i|}{\ln |p-i|}\right) & \text { if } 2 \mu(i)=v^{\prime}(i) \text { and } \lambda \kappa_{i} \neq 0, \\ v^{\prime}(i)-\mu(i)+O(|p-i|) & \text { if } 2|\mu(i)|>\left|v^{\prime}(i)\right| \text { and } \lambda \kappa_{i} \neq 0,\end{cases}
$$

as $p \rightarrow i$ for $i \in\{0,1\}$.

Proof. Recall that Lemma 3.2 describes the asymptotic behavior of $\pi(\cdot)$ as $p \rightarrow i$. From this we obtain

$$
\frac{v(p) \pi^{\prime}(p)}{\pi(p)}= \begin{cases}v^{\prime}(i) \frac{2 \mu(i)-v^{\prime}(i)}{v^{\prime}(i)}+O(|p-i|) & \text { if } 2|\mu(i)|<\left|v^{\prime}(i)\right| \text { or } \lambda \kappa_{i}=0, \\ \frac{v^{\prime}(i)}{\ln |p-i|}+O\left(\frac{|p-i|}{\ln |p-i|}\right) & \text { if } 2 \mu(i)=v^{\prime}(i) \text { and } \lambda \kappa_{i} \neq 0, \\ 0+O(|p-i|) & \text { if } 2|\mu(i)|>\left|v^{\prime}(i)\right| \text { and } \lambda \kappa_{i} \neq 0,\end{cases}
$$

as $p \rightarrow i$ for $i \in\{0,1\}$. Inserting this into

$$
\tilde{\mu}(p):=-\mu(p)+\frac{(v \pi)^{\prime}(p)}{\pi(p)}=-\mu(i)+v^{\prime}(i)+O(|p-i|)+\frac{v(p) \pi^{\prime}(p)}{\pi(p)}
$$

results in assertion (4.1).

Remark 4.1. Note that $\tilde{\mu}(0)<0$ if $\mu(0)>v^{\prime}(0)$, so that the diffusive motion of the timereversed process need not be confined to $[0,1]$. Nonetheless, because the boundary $p=0$ is inaccessible to the forward diffusion in this case (i.e. $2 \mu(0)>v^{\prime}(0)$ ), the fact that the process $\tilde{p}(\cdot)$ immediately jumps back into $(0,1)$ upon hitting 0 will ensure that the jump diffusion is confined to $[0,1]$.

We next show that if a boundary point is accessible to the forward jump diffusion $p(\cdot)$, either diffusively or via a jump, then it must be accessible to the backward diffusive motion governed by $\tilde{A}$. Recall the scale function $\tilde{S}$ from (2.3).

Lemma 4.2. Suppose that Assumption 2.1 holds. The boundary point $i \in\{0,1\}$ is accessible to the diffusive motion governed by $\tilde{A}$, that is, $\tilde{S}(i) \in \mathbb{R}$, if and only if $i$ is accessible to the forward jump diffusion $p(\cdot)$, that is, if $\lambda w_{i} \not \equiv 0$ or $|\mu(i)|<\left|v^{\prime}(i)\right|$.

Proof. Without loss of generality, we only prove the case $i=0$. According to Lemma 15.6.1 of [14], the boundary point 0 is accessible if and only if $\tilde{S}(0+)$ is finite. (This is a special case of Feller's boundary classification criteria.) Substituting the asymptotic expression for $\tilde{\mu}$ near $p=0$ (see Lemma 4.1) into the definition of $\tilde{S}$, we obtain, in the case $\lambda \kappa_{0}>0$,

$$
\tilde{S}^{\prime}(p):=\exp \left(-\int_{1 / 2}^{p} \frac{2 \tilde{\mu}(z)}{v(z)} \mathrm{d} z\right) \sim \begin{cases}p^{-2 \mu(0) / v^{\prime}(0)} & \text { if } 2|\mu(i)|<\left|v^{\prime}(i)\right|, \\ \frac{1}{p} \frac{1}{(\ln (p))^{2}} & \text { if } 2 \mu(i)=v^{\prime}(i), \\ p^{-\left(2 v^{\prime}(0)-2 \mu(0)\right) / v^{\prime}(0)} & \text { if } 2|\mu(i)|>\left|v^{\prime}(i)\right|,\end{cases}
$$

as $p \rightarrow 0$. In all three cases, $\tilde{S}^{\prime}(\cdot)$ is integrable over $\left(0, \frac{1}{2}\right]$. The case $\lambda \kappa_{0}=0$ follows from similar arguments. 
The following lemma shows that the rate constant $r_{i}$ (defined in (2.5)) is equal to $\infty$ if the boundary point $i \in\{0,1\}$ is inaccessible to the forward diffusive motion. Therefore, $\tilde{p}(\cdot)$ jumps whenever it hits $i$ as $\tilde{L}_{i}(t) \geq 0=R_{i}$. In addition, if there are no jumps in the forward process then $r_{i}=0$, and $\tilde{p}(\cdot)$ never jumps as $\tilde{L}_{i}(t)<\infty=R_{i}$.

Lemma 4.3. Suppose that Assumption 2.1 holds. Then the rate constant $r_{i}$ used to define the jump times of $\tilde{p}(\cdot)$ satisfies

$$
r_{i}:=\lim _{p \rightarrow i}\left(\frac{\tilde{m}(p)}{\pi(p)} \lambda \kappa_{i}\right) \begin{cases}\in(0, \infty) & \text { if } 2|\mu(i)|<\left|v^{\prime}(i)\right| \text { and } \lambda w_{i}(\cdot) \not \equiv 0 \\ =\infty & \text { if } 2|\mu(i)| \geq\left|v^{\prime}(i)\right| \text { and } \lambda w_{i}(\cdot) \not \equiv 0 \\ =0 & \text { if } \lambda w_{i}(\cdot) \equiv 0\end{cases}
$$

for $i \in\{0,1\}$.

Proof. Without loss of generality, we only prove the case $i=0$, as the case $i=1$ is similar. If $\lambda \kappa_{0}=0$ then $r_{0}=0$ is trivially correct. Assume that $\lambda \kappa_{0}>0$ for the rest of the proof. The asymptotic behavior of the scale density $\tilde{S}^{\prime}(\cdot)$ is given in (4.2). From this we derive the asymptotic behavior of the speed density $\tilde{m}(p)$ (defined in (2.3)) as $p \rightarrow 0$ :

$$
\tilde{m}(p)=\frac{2}{v(p) \tilde{S}^{\prime}(p)} \sim \begin{cases}p^{\left(2 \mu(0)-v^{\prime}(0)\right) / v^{\prime}(0)} & \text { if } 2 \mu(0)<v^{\prime}(0), \\ (\ln (p))^{2} & \text { if } 2 \mu(0)=v^{\prime}(0), \\ p^{\left(v^{\prime}(0)-2 \mu(0)\right) / v^{\prime}(0)} & \text { if } 2 \mu(0)>v^{\prime}(0) .\end{cases}
$$

Compare (4.4) with the boundary behavior of $\pi(\cdot)$ (see Lemma 3.2) to obtain (4.3).

\section{The generator of the time-reversed process}

In this section we identify the generator of the time-reversed process and show that this operator satisfies the duality condition given in (1.2). That this operator is also the generator of the jump diffusion process $\tilde{p}(\cdot)$ described in Section 2 will be established in the final two sections of the paper.

The following notation will be needed to formulate the generator of the time-reversed process. If $v(\mathrm{~d} p)=f(p) \mathrm{d} p$ is a measure on $[0,1]$ with continuous density $f:(0,1) \rightarrow(0, \infty)$ with respect to Lebesgue measure, then we write

$$
\left(D_{\nu} \psi\right)(p):=\lim _{y \rightarrow p} \frac{\psi(y)-\psi(p)}{\int_{p}^{y} f(q) \mathrm{d} q} \quad \text { for all } p \in(0,1)
$$

whenever this limit exists in $\mathbb{R}$ and denote by

$$
\begin{aligned}
& \mathfrak{D}\left(D_{\nu}\right):=\left\{\psi \in \mathcal{C}([0,1]): D_{\nu} \psi(p) \text { exists for all } p \in(0,1), D_{\nu} \psi\right. \text { is continuous } \\
& \text { in } \left.(0,1), D_{\nu} \psi(0+) \text { and } D_{\nu} \psi(1-) \text { exist in } \mathbb{R}\right\}
\end{aligned}
$$

the subset of functions which are mapped to continuous functions on $[0,1]$. Note that $\psi \in$ $\mathcal{C}^{1}((0,1))$ and $D_{v} \psi(p)=\psi^{\prime}(p) / f(p), p \in(0,1)$, for every $\psi \in \mathfrak{D}\left(D_{v}\right)$. For $\psi \in \mathfrak{D}\left(D_{v}\right)$, the definition of $D_{v}$ extends to the boundary via

$$
D_{\nu} \psi(0):=D_{\nu} \psi(0+) \quad \text { and } \quad D_{\nu} \psi(1):=D_{\nu} \psi(1-) .
$$


Using this notation, the generator $\tilde{A}$ of the backward diffusive motion reads as

$$
\begin{aligned}
(\tilde{A} \psi)(p) & =\frac{1}{2} v(p) \psi^{\prime \prime}(p)+\tilde{\mu}(p) \psi^{\prime}(p) \\
& =\frac{1}{2} v(p) \tilde{S}^{\prime}(p)\left(\frac{1}{\tilde{S}^{\prime}} \psi^{\prime \prime}+\frac{1}{\tilde{S}^{\prime}} \frac{2 \tilde{\mu}}{v} \psi^{\prime}\right)(p) \\
& =D_{\tilde{m}} D_{\tilde{S}} \psi(p)
\end{aligned}
$$

for every $\psi \in \mathcal{C}^{2}([0,1])$. The following set will be a core for the generator of the time-reversed process:

$$
\begin{aligned}
\mathbb{H}:=\left\{\psi \in \mathfrak{D}\left(D_{\tilde{S}}\right): D_{\tilde{S}} \psi \in \mathfrak{D}\left(D_{\tilde{m}}\right) \text { and, for } i \in\{0,1\},\right. \\
\left.\lim _{p \rightarrow i}\left(\frac{1}{2} v \pi \psi^{\prime}\right)(p)=(-1)^{i+1} \int_{0}^{1}(\psi(p)-\psi(i)) \lambda w_{i}(p) \pi(p) \mathrm{d} p\right\} .
\end{aligned}
$$

The following lemma asserts that the restriction of $D_{\tilde{m}} D_{\tilde{S}}$ to $\mathbb{H}$ extends to a strong generator of a Markov process. Indeed, this can be deduced from Theorem II.4 of [19], which shows that the restriction of $D_{\tilde{m}} D_{\tilde{S}}$ to

$$
\begin{aligned}
\left\{\psi \in \mathfrak{D}\left(D_{\tilde{S}}\right): D_{\tilde{S}} \psi \in\right. & \mathfrak{D}\left(D_{\tilde{m}}\right) \text { and, if } i \in\{0,1\} \text { is accessible, } \\
& \left.\chi_{i} \psi(i)+\int_{0}^{1} \frac{\psi(i)-\psi(p)}{\left|p_{i}(i)-p_{i}(p)\right|} \mathrm{d} q_{i}(p)+\eta_{i} D_{\tilde{m}} D_{\tilde{S}} \psi(i)=0\right\}
\end{aligned}
$$

is the strong generator of a Feller semigroup if $\chi_{i}$ and $\eta_{i}$ are both nonnegative, if $q_{i}$ is a nondecreasing function on $[0,1]$, and if $p_{i}$ is continuous, nondecreasing, and equal to $\tilde{S}$ in a neighborhood of $i, i=0,1$. Only the case $0=\chi_{0}=\chi_{1}=\eta_{0}=\eta_{1}=q(1-)-q(0+) \neq$ $q(1)-q(0)$ is excluded. The quotient in (5.2) is to be interpreted as $(-1)^{i+1} D_{\tilde{S}} \psi(i)$ for $p=i \in\{0,1\}$ and the integral with respect to $\mathrm{d} q_{i}(p)$ denotes the Lebesgue-Stiltjes integral with respect to $q_{i}$.

Lemma 5.1. Suppose that Assumption 2.1 holds. The restriction of $D_{\tilde{m}} D_{\tilde{S}}$ to the set $\mathbb{H}$ extends to a strong generator of a Markov process.

Proof. By Theorem II.4 of [19], it suffices to prove that $\mathbb{H}$ is of the form (5.2). According to Lemma 4.2 , the boundary point $i \in\{0,1\}$ is accessible to the diffusion governed by $D_{\tilde{m}} D_{\tilde{S}}$ if and only if $\lambda w_{i}(\cdot) \not \equiv 0$ or $|\mu(i)|<\left|v^{\prime}(i)\right|$. First we show that the condition in (5.1) is trivial if $i$ is inaccessible, that is, we show that $\lim _{p \rightarrow i}\left(v \pi \psi^{\prime}\right)(p)=0$ for every $\psi \in \mathfrak{D}\left(D_{\tilde{S}}\right)$. Suppose that

$$
C:=\lim _{p \rightarrow i}\left(v \pi \psi^{\prime}\right)(p)>0
$$

for some function $\psi \in \mathfrak{D}\left(D_{\tilde{S}}\right)$. By Lemma 3.2, $\pi(p)$ is bounded above by $-\bar{C} \ln (|p-i|)$ in a neighborhood of $i$ for some $\bar{C}>0$. Thus, in a neighborhood of $i$,

$$
\psi^{\prime}(p) \geq \frac{-C}{2 v(p) \pi(p)} \geq \frac{C}{4 \bar{C}\left|v^{\prime}(i)\right||p-i| \ln (|p-i|)} .
$$

Integrating over $\left[\frac{1}{2}, p\right]$ implies that $\psi(p)$ is bounded below by $C \ln (-\ln (|p-i|)) /\left(4 \bar{C}\left|v^{\prime}(i)\right|\right)$ as $p \rightarrow i$, which contradicts $\psi \in \mathcal{C}(I)$. An analogous argument applies to the case $C<0$. 
Now, let $i$ be accessible to the forward process, that is, $\lambda \kappa_{i}>0$ or $|\mu(i)|<\left|v^{\prime}(i)\right|$. Note that $\tilde{S}(\cdot)$ is a bounded, continuous, nondecreasing function in a neighborhood of $i$. Choose $\chi_{i}, \eta_{i}=0$ and $p_{i}(p):=\tilde{S}(p)$. Furthermore, let

$$
q_{i}(p):=\int_{0}^{p}\left|p_{i}(i)-p_{i}(y)\right| w_{i}(y) \pi(y) \mathrm{d} y+c_{0} \mathbf{1}_{(0,1]}(p) \mathbf{1}_{\{i=0\}}+c_{1} \mathbf{1}_{1}(p) \mathbf{1}_{\{i=1\}},
$$

where $c_{0}, c_{1} \in[0, \infty)$ are to be chosen later. Note that $q_{i}$ is bounded and that $\mathrm{d} q_{i}$ puts mass $c_{i}$ on the point $i$. With these definitions, the condition in (5.2) takes the form

$$
\int_{0}^{1}(\psi(i)-\psi(p)) w_{i}(p) \pi(p) \mathrm{d} p=(-1)^{i} D_{\tilde{S}} \psi(i) c_{i} .
$$

It remains to choose $c_{i} \in[0, \infty)$ such that

$$
D_{\tilde{S}} \psi(i) c_{i}=\lim _{p \rightarrow i}\left(\frac{1}{2} v \pi \psi^{\prime}\right)(p)
$$

for every $\psi \in \mathbb{H}$. Using the boundary behavior (4.2) of $\tilde{S}(\cdot)$ and the asymptotic behavior (3.4) of $\tilde{\pi}(\cdot)$, we arrive at

$$
\begin{aligned}
\left(v \pi \psi^{\prime}\right)(p) & \sim|p-i| \pi(p) \tilde{S}^{\prime}(p) D_{\tilde{S}} \psi(p) \\
& \sim \begin{cases}D_{\tilde{S}} \psi(p) & \text { if } 2|\mu(i)|<\left|v^{\prime}(i)\right|, \\
D_{\tilde{S}} \psi(p) \frac{-1}{\ln (|p-i|)} & \text { if } 2 \mu(i)=v^{\prime}(i), \\
D_{\tilde{S}} \psi(p)|p-i|^{2 \mu(i) / v^{\prime}(i)-1} & \text { if } 2|\mu(i)|>\left|v^{\prime}(i)\right|,\end{cases}
\end{aligned}
$$

as $p \rightarrow i$. This shows that (5.3) holds with some constant $c_{i} \in[0, \infty)$. This completes the proof.

Lemma 5.2. Suppose that Assumption 2.1 holds. Let the process $(p(t): t \geq 0)$ be in equilibrium. Then the time-reversed process $\left(\bar{p}_{t}: t \geq 0\right)$ exists, that is, there exists a process $\left(\bar{p}_{t}: t \geq 0\right)$ satisfying

$$
(\bar{p}(t): t \leq T) \stackrel{\mathrm{D}}{=}(p(T-t): t \leq T) \text { for all } T \geq 0 .
$$

In addition, $\mathbb{H}$ is a core for the generator $\tilde{G}$ of $\left(\bar{p}_{t}: t \geq 0\right)$ and

$$
\tilde{G} \psi=\frac{1}{2} v \psi^{\prime \prime}+\tilde{\mu} \psi^{\prime}=D_{\tilde{m}} D_{\tilde{S}} \psi \quad \text { for all } \psi \in \mathbb{H} .
$$

Proof. Let $\tilde{G}$ be the closure of the operator defined in (5.4). By Lemma 5.1, $\tilde{G}$ is the strong generator of a Markov process $\left(\bar{p}_{t}: t \geq 0\right)$. Recall the generator $G$ of $p(\cdot)$ from (2.1). We will prove that $\tilde{G}$ is the adjoint operator of $G$ with respect to the invariant measure $\pi(p) \mathrm{d} p$. Let $0<\varepsilon<\frac{1}{2}, \phi \in \mathcal{C}^{2}([0,1])$, and $\psi \in \mathbb{H}$. The functions $v, \mu, \phi, \psi$, and $\pi$ are $\mathcal{C}^{2}$ in $[\varepsilon, 1-\varepsilon]$. Integration by parts yields

$$
\begin{aligned}
\int_{\varepsilon}^{1-\varepsilon} & G \phi \psi \pi \mathrm{d} p-\phi(0) \int_{\varepsilon}^{1-\varepsilon} \lambda w_{0} \psi \pi \mathrm{d} p-\phi(1) \int_{\varepsilon}^{1-\varepsilon} \lambda w_{1} \psi \pi \mathrm{d} p \\
= & \int_{\varepsilon}^{1-\varepsilon}\left[\phi^{\prime \prime} \cdot\left(\frac{1}{2} v \psi \pi\right)+\phi^{\prime} \cdot(\mu \psi \pi)-\lambda \phi \psi \pi\right] \mathrm{d} p \\
= & {\left[\phi^{\prime} \frac{1}{2} v \psi \pi\right]_{\varepsilon}^{1-\varepsilon}+\left[\phi\left(\mu \psi \pi-\left(\frac{1}{2} v \psi \pi\right)^{\prime}\right)\right]_{\varepsilon}^{1-\varepsilon} } \\
& +\int_{\varepsilon}^{1-\varepsilon} \phi\left[\left(\frac{1}{2} v \psi \pi\right)^{\prime \prime}-(\mu \psi \pi)^{\prime}-\lambda \psi \pi\right] \mathrm{d} p
\end{aligned}
$$


As $\pi$ satisfies (3.1a), we see that

$$
\left(\frac{1}{2} v \psi \pi\right)^{\prime \prime}-(\mu \psi \pi)^{\prime}-\lambda \psi \pi=\frac{1}{2} v \psi^{\prime \prime} \pi+\left(\frac{(v \pi)^{\prime}}{\pi}-\mu\right) \psi^{\prime} \pi=D_{\tilde{m}} D_{\tilde{S}} \psi \pi .
$$

The functions $G \phi, \psi, w_{0}, w_{1}, \phi$, and $D_{\tilde{m}} D_{\tilde{S}} \psi$ are bounded and $\pi$ is integrable. Hence, we may apply the dominated convergence theorem to the integrals in (5.5) as $\varepsilon \rightarrow 0$. This also proves that the limits of the boundary terms in (5.5) exist as $\varepsilon \rightarrow 0$. Thus, letting $\varepsilon \rightarrow 0$ in (5.5), we obtain

$$
\begin{aligned}
\int_{0}^{1} G \phi \psi \pi \mathrm{d} p-\int_{0}^{1} \phi D_{\tilde{m}} D_{\tilde{S}} \psi \pi \mathrm{d} p \\
=\phi(1)\left(\psi(1)\left(\mu \pi-\left(\frac{1}{2} v \pi\right)^{\prime}\right)(1-)-\lim _{p \rightarrow 1}\left(\frac{1}{2} v \pi \psi^{\prime}\right)(p)+\int_{0}^{1} \lambda w_{1} \psi \pi \mathrm{d} p\right) \\
\quad-\phi(0)\left(\psi(0)\left(\mu \pi-\left(\frac{1}{2} v \pi\right)^{\prime}\right)(0+)-\lim _{p \rightarrow 0}\left(\frac{1}{2} v \pi \psi^{\prime}\right)(p)-\int_{0}^{1} \lambda w_{0} \psi \pi \mathrm{d} p\right) \\
=0
\end{aligned}
$$

for all $\phi \in \mathcal{C}^{2}$ and $\psi \in \mathbb{H}$. The last equality follows from (3.1b)-(3.1d) and the fact that $\psi \in \mathbb{H}$. This proves that $G$ and $\tilde{G}$ are adjoint to each other. Consequently, the semigroups of $(p(t): t \geq 0)$ and of $\left(\bar{p}_{t}: t \geq 0\right)$ are adjoint to each other. According to Nelson [23], this implies that the Markov process $(\bar{p}(t): t \geq 0)$ associated with $\tilde{G}$ has the same law as the time-reversed process of $(p(t): t \geq 0)$. This completes the proof.

\section{The local-time process}

In this section we describe some properties of the local-time process of $\tilde{p}(\cdot)$. First we show existence. Recall the scale function $\tilde{S}$ and the speed measure $\tilde{m}$ from (2.3).

Lemma 6.1. Suppose that Assumption 2.1 holds. Then there exists a unique, nonnegative process

$$
\left(\tilde{L}_{p}(t): t \geq 0, p \in[0,1]\right)
$$

which is a.s. continuous in $(t, p)$ and which satisfies

$$
\int_{0}^{t} f(\tilde{p}(u)) \mathrm{d} u=\int_{0}^{1} f(p) \tilde{L}_{p}(t) \tilde{m}(\mathrm{~d} p) \quad \text { for all } t \geq 0,
$$

for all measurable $f:[0,1] \rightarrow[0, \infty)$ a.s. In addition, if $2|\mu(i)| \geq\left|v^{\prime}(i)\right|$ for $i \in\{0,1\}$ then $\tilde{L}_{i}(\cdot) \equiv 0$ a.s.

Proof. Let $0=: \tau_{0} \leq \tau_{1}<\tau_{2}<\cdots$ be the jump times of $\tilde{p}(\cdot)$. Then, by construction of $\tilde{p}(\cdot),\left(\tilde{p}\left(t+\tau_{n-1}\right): 0 \leq t<\tau_{n}-\tau_{n-1}\right), n \in \mathbb{N}_{\geq 1}$, are independent diffusions governed by $\tilde{A}$. It is well known that $\tilde{p}\left(\cdot+\tau_{n-1}\right)$ can be written in terms of a Brownian motion as follows. Let $\left\{B^{y, n}: y \in \mathbb{R}, n \in \mathbb{N}\right\}$ be a family of independent standard Brownian motions with $B_{0}^{y, n}=y$. Denote by $\left(L_{x}^{B^{y, n}}(t): t \geq 0, x \in \mathbb{R}\right)$ the local-time process of $B{ }^{y, n}$ (see, e.g. Section 2.8 of [11]), and define

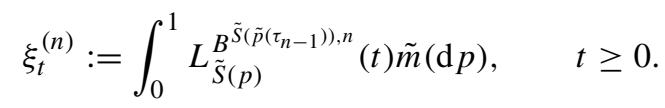


Then a version of $\tilde{p}\left(\cdot+\tau_{n-1}\right)$ is given by

$$
\tilde{p}\left(t+\tau_{n-1}\right)=\tilde{S}^{-1}\left(\begin{array}{c}
\tilde{S}\left(\tilde{p}\left(\tau_{n-1}\right)\right), n \\
\left(\xi_{t}^{(n)}\right)^{-1}
\end{array}\right), \quad 0 \leq t<\tau_{n}-\tau_{n-1} .
$$

Inserting this into the occupation time formula of the Brownian motion, a short calculation (see, e.g. Section 5.4 of [11]) shows that

$$
\int_{0}^{t} f\left(\tilde{p}\left(r+\tau_{n-1}\right)\right) \mathrm{d} r=\int_{0}^{1} f(p) \hat{L}_{p}^{(n)}(t) \tilde{m}(\mathrm{~d} p), \quad 0 \leq t<\tau_{n}-\tau_{n-1},
$$

where the local-time process of $\tilde{p}\left(\cdot+\tau_{n-1}\right)$ with respect to the speed measure is

$$
\hat{L}_{p}^{(n)}(t):=L_{\tilde{S}(p)}^{B^{\tilde{S}\left(\tilde{p}\left(\tau_{n-1}\right)\right), n}}\left(\left(\xi_{t}^{(n)}\right)^{-1}\right), \quad 0 \leq t<\tau_{n}-\tau_{n-1}, p \in[0,1], n \in \mathbb{N}_{\geq 1} .
$$

Now we put the independent path segments together by defining

$$
\tilde{L}_{p}(t):=\hat{L}_{p}^{(n)}\left(t-\tau_{n-1}\right)+\sum_{k=1}^{n-1} \hat{L}_{p}^{(k)}\left(\tau_{k}-\tau_{k-1}\right) \quad \text { if } \tau_{n-1} \leq t<\tau_{n} .
$$

It is easy to use (6.2) to show that $\left(\tilde{L}_{p}(t): t \geq 0, p \in[0,1]\right)$ satisfies (6.1). Uniqueness follows from standard arguments.

If $2|\mu(i)| \geq\left|v^{\prime}(i)\right|$ then $\tilde{p}(\cdot)$ jumps into $(0,1)$ as soon as it hits the boundary and we conclude that $\tilde{p}(t) \neq i$ for all $t \geq 0$. Thus, the local time at this boundary point is identically zero. This completes the proof.

In the next section, we will need to be able control the second moment of the local time of the time-reversed jump diffusion at a boundary point. We first prove the following estimate concerning the local time of a standard Brownian motion.

Lemma 6.2. Let $\varepsilon, \delta>0$, and let $\left(B_{t}: t \geq 0\right)$ be a standard Brownian motion with local time $L_{x}^{B}(\cdot)$ at $x \in \mathbb{R}$. Suppose that the function $\bar{S}:[0, \delta] \rightarrow \mathbb{R}$ is nondecreasing. Define $\Delta:=\bar{S}(\delta)-\bar{S}(0)$ and

$$
\zeta_{t}:=\int_{0}^{\delta} \frac{1}{\varepsilon} L_{\bar{S}(y)}^{B}(t) \mathrm{d} y, \quad t \geq 0 .
$$

Then, for each $m>0$, there exists a constant $C_{m}$ independent of $\varepsilon$ and of $\delta$ such that

$$
\mathrm{E}^{\bar{S}(p)}\left[\left(L_{\bar{S}(p)}^{B}\left(\zeta_{t}^{-1}\right)\right)^{m}\right] \leq C_{m}(\varepsilon t)^{m / 2}\left[\left(\frac{\varepsilon t}{\Delta^{2}}\right)^{m / 2}+1\right]
$$

for all $p \in[0, \delta]$ and $t \geq 0$.

Proof. Inequality (6.4) is trivial if $\Delta=0$ or $t=0$, so we may and will assume that $\Delta>0$ and $t>0$. Fix $p \in[0, \delta]$. The left-hand side of (6.4) does not depend on the value of $\bar{S}(p)$, so we will also assume without loss of generality that $\bar{S}(p)=0$. Denote by $B_{t}^{*}:=\max \left\{B_{s}: s \leq t\right\}$ and by $|B|_{t}^{*}:=\max \left\{\left|B_{s}\right|: s \leq t\right\}$ the process of the maximum and the process of the absolute maximum, respectively. Define $Z_{t}:=\bar{S}^{-1}\left(B_{\zeta_{t}^{-1}}\right)$ for $t \geq 0$. According to Section 5.4 of [11], $L_{p}^{Z}(t):=L_{0}^{B}\left(\zeta_{t}^{-1}\right)$ is the local time of $\left(Z_{t}: t_{t} \geq 0\right)$ at $p$. The process $\left(Z_{t}: t \geq 0\right)$ is equal in distribution to the process $\left(\bar{S}^{-1}\left(B_{\varepsilon t}\right): t \geq 0\right)$ reflected at 0 and at $\delta$. Another way to construct $\left(\bar{S}\left(Z_{t}\right): t \geq 0\right)$ is to take the path of $\left(B_{\varepsilon t}: t \geq 0\right)$ and to identify each $x \in[\bar{S}(0), \bar{S}(\delta)]$ with 
the set $\{x+2 \Delta z, 2 \bar{S}(\delta)-x+2 \Delta z: z \in \mathbb{Z}\}$. Thus, the local time $L_{p}^{Z}(t)$ of $\left(Z_{t}: t \geq 0\right)$ in $p$ is equal in distribution to the sum of $L_{2 \Delta z}^{B}(\varepsilon t)+L_{2 \Delta z+2 \bar{S}(\delta)}^{B}(\varepsilon t)$ over $z \in \mathbb{Z}$. Note that $L_{x}^{B}(\varepsilon t)=0$ a.s. on the event $\left\{|B|_{\varepsilon t}^{*}<|x|\right\}, x \in \mathbb{R}$. In addition, note that convexity of $0 \leq x \mapsto x^{m}$ implies that $k^{m-1}\left(a_{1}+\cdots+a_{k}\right)^{m} \leq\left(a_{1}^{m}+\cdots+a_{k}^{m}\right)$ for $a_{1}, \ldots, a_{k} \geq 0, k \in \mathbb{N}$. Therefore,

$$
\begin{aligned}
& \mathrm{E}^{p}\left[\left(L_{p}^{Z}(t)\right)^{m}\right] \\
& \quad=\mathrm{E}^{0}\left[\sum_{k \in 2 \Delta \mathbb{N}_{\geq 0}} \mathbf{1}_{[k, k+2 \Delta)}\left(|B|_{\varepsilon t}^{*}\right)\left(\sum_{z=-k / 2 \Delta}^{k / 2 \Delta} \sum_{x \in\{0,2 \bar{S}(\delta)\}} L_{2 \Delta z+x}^{B}(\varepsilon t)\right)^{m}\right] \\
& \quad \leq \mathrm{E}^{0}\left[\sum_{k \in 2 \Delta \mathbb{N}_{\geq 0}} \mathbf{1}_{[k, k+2 \Delta)}\left(|B|_{\varepsilon t}^{*}\right)\left(\frac{2 k}{\Delta}+2\right)^{m-1} \sum_{z=-k / 2 \Delta}^{k / 2 \Delta} \sum_{x \in\{0,2 \bar{S}(\delta)\}}\left(L_{2 \Delta z+x}^{B}(\varepsilon t)\right)^{m}\right] .
\end{aligned}
$$

Use the strong Markov property (see, e.g. Proposition 2.6.17 of [13]) to restart the Brownian motion at the first hitting times of $2 \Delta z$ and $2 \Delta z+2 \bar{S}(\delta)$, respectively. Thus, the left-hand side of (6.5) is bounded above by

$$
\begin{aligned}
& \sum_{k \in 2 \Delta \mathbb{N}_{\geq 0}} \mathrm{P}^{0}\left(|B|_{\varepsilon t}^{*} \in[k, k+2 \Delta)\right)\left(\frac{2 k}{\Delta}+2\right)^{m-1} 2 \sum_{i=-k / 2 \Delta}^{k / 2 \Delta} \mathrm{E}^{0}\left[\left(L_{0}^{B}(\varepsilon t)\right)^{m}\right] \\
& \leq \mathrm{E}^{0}\left[\left(\frac{2|B|_{\varepsilon t}^{*}}{\Delta}+2\right)^{m}\right] \mathrm{E}^{0}\left[\left(L_{0}^{B}(\varepsilon t)\right)^{m}\right] .
\end{aligned}
$$

Note that $2 L_{0}^{B}(t)$ and $B_{t}^{*}$ are equal in distribution; see, e.g. Theorem 3.6.17 of [13]. Therefore, the left-hand side of (6.5) is bounded above by

$$
2^{m}\left[\mathrm{E}^{0}\left[\left(\frac{|B|_{\varepsilon t}^{*}}{\Delta}\right)^{m}\right]+1\right] \mathrm{E}^{0}\left[\left(\frac{1}{2} B_{\varepsilon t}^{*}\right)^{m}\right] \leq\left[K_{m / 2}\left(\frac{\varepsilon t}{\Delta^{2}}\right)^{m / 2}+1\right] K_{m / 2}(\varepsilon t)^{m / 2},
$$

where $K_{m / 2} \geq 1$ is a suitable constant which is independent of $\Delta, \varepsilon$, and $t$. The last step follows from the Burkholder-Davis-Gundy inequality; see, e.g. Theorem 3.3.28 of [13]. Therefore, (6.4) holds with $C_{m}:=K_{m / 2}^{2}$. This completes the proof.

In the proof of Theorem 2.1, we will need to exploit the fact that, in the $L^{2}$ sense, the local time $\left(\tilde{L}_{i}(t): t \geq 0\right)$ at a boundary point $i \in\{0,1\}$ of the backward process started at $i$ decreases to 0 faster than $\sqrt{t}$ as $t \rightarrow 0$. This might be surprising as we can show that

$$
\mathrm{E}^{0}\left[\left(L_{0}^{B}(t)\right)^{2}\right] \sim t \quad \text { as } t \rightarrow 0 .
$$

However, the infinitesimal variance $v(\cdot)$ is 0 in $i$. Thus, informally speaking, the diffusion governed by $\tilde{A}$ is pushed away from 0 almost deterministically at rate $\tilde{\mu}(i)>0$ if the boundary point is accessible at all.

Lemma 6.3. Suppose that Assumption 2.1 holds. Then the local time at the boundary satisfies

$$
\lim _{t \rightarrow 0} \frac{1}{t} \mathrm{E}^{i}\left[\left(\tilde{L}_{i}(t)\right)^{2}\right]=0
$$

for $i \in\{0,1\}$. 
Proof. If $2|\mu(i)| \geq\left|v^{\prime}(i)\right|$ then Lemma 6.1 tells us that $\tilde{L}_{i}(t)=0$, which implies the assertion in this case. For the rest of the proof, assume that $2|\mu(i)|<\left|v^{\prime}(i)\right|$. Without loss of generality, we only prove the case $i=0$, as the case $i=1$ is similar. To begin, we prove that (6.6) holds with $\tilde{L}_{0}(\cdot)$ replaced by $\hat{L}_{0}^{(1)}(\cdot)$. For $\delta \in(0,1)$, define

$$
\underline{\tilde{m}}(\delta)=\inf _{x \leq \delta} \tilde{m}(x) .
$$

The asymptotic behavior (4.4) of $\tilde{m}(\cdot)$ implies that $\lim _{\delta \rightarrow 0} \underline{\tilde{m}}(\delta)=\lim _{p \rightarrow 0} \tilde{m}(p)=\infty$. Recall that $B^{y, 1}$ is a standard Brownian motion started at $B_{0}^{y, 1}=y$. Observe that

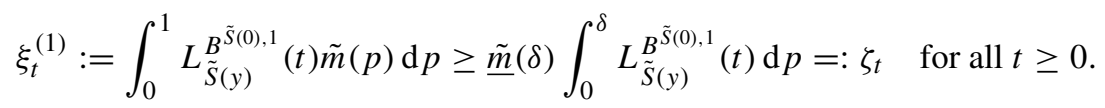

Using $\left(\xi_{t}^{(1)}\right)^{-1} \leq \zeta^{-1}$, we obtain an upper bound for $\hat{L}_{0}^{(1)}(\cdot)$ as follows:

$$
\begin{aligned}
\frac{1}{t} \mathrm{E}^{0}\left[\left(\hat{L}_{0}^{(1)}(t)\right)^{2}\right] & =\frac{1}{t} \mathrm{E}\left[\left(L_{\tilde{S}(0)}^{B^{\tilde{S}(0)}, 1}\left(\left(\xi_{t}^{(1)}\right)^{-1}\right)\right)^{2}\right] \\
& \leq \frac{1}{t} \mathrm{E}\left[\left(L_{\tilde{S}(0)}^{\left.\left.B^{\tilde{S}(0), 1}\left(\zeta_{t}^{-1}\right)\right)^{2}\right]}\right.\right. \\
& \leq \frac{C_{2}}{\underline{\tilde{m}}(\delta)}\left(\frac{t}{\underline{\tilde{m}}(\delta)(\tilde{S}(\delta)-\tilde{S}(0))^{2}}+1\right) \\
& \stackrel{t \rightarrow 0}{\longrightarrow} \frac{C_{2}}{\tilde{\tilde{m}}(\delta)} \\
& \stackrel{\delta \rightarrow 0}{\longrightarrow} 0
\end{aligned}
$$

for some constant $C_{2}$ which is independent of $t$ and $\delta$. The last inequality is Lemma 6.2.

Now we come to the local-time process $\tilde{L}_{0}(\cdot)$. Recall $r_{0}$ and $R_{0}$ from Section 2 , and let $\tau_{1}$ be the first jump time of $\tilde{p}(\cdot)$ from the boundary point 0 . The local time $\hat{L}_{0}(t)$ converges to 0 a.s. as $t \rightarrow 0$. By the theorem of dominated convergence, this implies that $\mathrm{E}^{0}\left[\hat{L}_{0}(t)\right] \rightarrow 0$ as $t \rightarrow 0$. Thus, there exists a $t_{0} \geq 0$ such that $r_{0} \mathrm{E}^{0}\left[\hat{L}_{0}^{(1)}(t)\right] \leq \frac{1}{4}$ for all $t \leq t_{0}$. Then we obtain, from the definition of $\tilde{L}_{0}(\cdot),(6.3)$, and the Markov property,

$$
\begin{aligned}
\mathrm{E}^{0}\left[\left(\tilde{L}_{0}(t)\right)^{2} \mathbf{1}_{\left\{\tau_{1}<t\right\}}\right] & \leq 2 \mathrm{E}^{0}\left[\left(\hat{L}_{0}^{(1)}(t)\right)^{2}\right]+2 \mathrm{E}^{0}\left[\left(\tilde{L}_{0}(t)-\hat{L}_{0}^{(1)}(t)\right)^{2} \mathbf{1}_{\left\{\hat{L}_{0}^{(1)}(t) \geq R_{0}\right\}}\right] \\
& \leq 2 \mathrm{E}^{0}\left[\left(\hat{L}_{0}^{(1)}(t)\right)^{2}\right]+2 \mathrm{E}^{\mathcal{L}\left(\tilde{p}\left(\tau_{1}\right)\right)}\left[\left(\tilde{L}_{0}(t)\right)^{2}\right] \mathrm{E}^{0}\left[1-\mathrm{e}^{-r_{0} \hat{L}_{0}^{(1)}(t)}\right] \\
& \leq 2 \mathrm{E}^{0}\left[\left(\hat{L}_{0}^{(1)}(t)\right)^{2}\right]+2 \mathrm{E}^{0}\left[\left(\tilde{L}_{0}(t)\right)^{2}\right] \frac{1}{4}
\end{aligned}
$$

for all $t \leq t_{0}$. Using this estimate, we get, for $t \leq t_{0}$,

$$
\begin{aligned}
\mathrm{E}^{0}\left[\left(\tilde{L}_{0}(t)\right)^{2}\right] & \leq \mathrm{E}^{0}\left[\left(\hat{L}_{0}^{(1)}(t)\right)^{2}\right]+\mathrm{E}^{0}\left[\left(\tilde{L}_{0}(t)\right)^{2} \mathbf{1}_{\left\{\tau_{1}<t\right\}}\right] \\
& \leq 3 \mathrm{E}^{0}\left[\left(\hat{L}_{0}^{(1)}(t)\right)^{2}\right]+\frac{1}{2} \mathrm{E}^{0}\left[\left(\tilde{L}_{0}(t)\right)^{2}\right] .
\end{aligned}
$$

Therefore,

$$
\lim _{t \rightarrow 0} \frac{1}{t} \mathrm{E}^{0}\left[\left(\tilde{L}_{0}(t)\right)^{2}\right] \leq 6 \lim _{t \rightarrow 0} \frac{1}{t} \mathrm{E}^{0}\left[\left(\hat{L}_{0}^{(1)}(t)\right)^{2}\right]=0,
$$

where the last equality is (6.7). This completes the proof. 


\section{The backward process}

In Section 5, we identified the generator $\tilde{G}$ of the time-reversed process (see Lemma 5.2). However, while it is clear that the boundary behavior of this process must be prescribed by the domain of the generator, it is difficult to see how a qualitative description of the process can be deduced from the analytical condition that defines this domain. To address this issue, we show in this section that the process $\tilde{p}(\cdot)$ defined in Section 2 also has generator $\tilde{G}$. This confirms the heuristic arguments given in the introduction and shows that the time-reversed process is a jump diffusion process whose jump times depend on the local-time process constructed in the preceding section.

The proof of Theorem 2.1 is based on the Itô-Tanaka formula for semimartingales, which involves the semimartingale local-time process. Because this local time differs by a scalar factor from the local-time process introduced in Section 6 (see (7.4) below), we have restated the semimartingale Itô-Tanaka formula in terms of $\tilde{L}_{p}(\cdot)$. This is done in the following lemma.

Lemma 7.1. Suppose that Assumption 2.1 holds. Let $(\tilde{Y}(t): t \geq 0)$ be a diffusion corresponding to the generator $\tilde{A}$ defined in (2.2). Then, for each $\psi \in \mathbb{H}$,

$$
\begin{aligned}
\psi(\tilde{Y}(t))-\psi(\tilde{Y}(0))= & \int_{0}^{t} D_{\tilde{m}} D_{\tilde{S}} \psi(\tilde{Y}(u)) \mathrm{d} u+\int_{0}^{t} \psi^{\prime}\left(\tilde{Y}_{u}\right) \sqrt{v\left(\tilde{Y}_{u}\right)} \mathrm{d} B_{u} \\
& +\frac{1}{2} \tilde{L}_{0}(t) D_{\tilde{S}} \psi(0)-\frac{1}{2} \tilde{L}_{1}(t) D_{\tilde{S}} \psi(1)
\end{aligned}
$$

for all $t \geq 0$ a.s.

Proof. Fix $\psi \in \mathbb{H}$. We approximate $\psi$ with suitable functions and apply the semimartingale Itô-Tanaka formula. Denote by $\left(\bar{L}_{p}(t)\right)_{t \geq 0, p \in[0,1]}$ the semimartingale local-time process of $(\tilde{Y}(t): t \geq 0)$. We remark that, in general, this local-time process is distinct from the local time, $\tilde{L}_{p}(\cdot)$, introduced in the preceding section. By Theorem 3.7.1 of [13] we may and we will assume that $\bar{L}_{p}(t)$ is continuous in $t$ and càdlàg in $p$. The occupation time formula (Theorem 3.7.1 of [13]) states that

$$
\int_{0}^{t} \psi(\tilde{Y}(u)) v(\tilde{Y}(u)) \mathrm{d} u=2 \int_{0}^{1} \psi(p) \bar{L}_{p}(t) \mathrm{d} p, \quad t \geq 0,
$$

a.s. Let $f$ be a continuous function which is $\mathcal{C}^{2}$ except in $\left\{a_{1}, \ldots, a_{n}\right\} \subset[0,1]$, and which admits finite limits $f^{\prime}\left(a_{k}+\right)$ and $f^{\prime}\left(a_{k}-\right), k=1, \ldots, n$. Then the Itô-Tanaka formula for continuous semimartingales (see Theorem 3.7.1 and Problem 3.6.24 of [13]) states that

$$
\begin{aligned}
f\left(\tilde{Y}_{t}\right)-f\left(\tilde{Y}_{0}\right)= & \int_{0}^{t} f^{\prime}\left(\tilde{Y}_{u}\right) \tilde{\mu}\left(\tilde{Y}_{u}\right) \mathrm{d} u+\int_{0}^{t} f^{\prime \prime}\left(\tilde{Y}_{u}\right) \frac{1}{2} v\left(\tilde{Y}_{u}\right) \mathrm{d} u+\int_{0}^{t} f^{\prime}\left(\tilde{Y}_{u}\right) \sqrt{v\left(\tilde{Y}_{u}\right)} \mathrm{d} B_{u} \\
& +\sum_{k=1}^{n} \bar{L}_{a_{k}}(t)\left[f^{\prime}\left(a_{k}+\right)-f^{\prime}\left(a_{k}-\right)\right] \quad \text { a.s. }
\end{aligned}
$$

For every $n \in \mathbb{N}$, let $\psi_{n}$ be a continuous function which is equal to $\psi$ in $(1 / n, 1-1 / n)$ and which is constant both in $[0,1 / n]$ and in $[1-1 / n, 1]$. In addition, suppose that $\left(\psi_{n}\right)_{n \in \mathbb{N}}$ approximates $\psi$ uniformly in $[0,1]$ and that $\left(D_{\tilde{m}} D_{\tilde{S}} \psi_{n}\right)_{n \in \mathbb{N}}$ approximates $D_{\tilde{m}} D_{\tilde{S}} \psi$ pointwise and boundedly in $(0,1)$. Note that

$$
D_{\tilde{S}} \psi_{n}\left(\frac{1}{n}-\right)=0 \quad \text { and } \quad D_{\tilde{S}} \psi_{n}\left(\frac{1}{n}+\right)=D_{\tilde{S}} \psi\left(\frac{1}{n}\right) .
$$


Comparing the occupation time formula (7.2) of $\bar{L}_{p}(\cdot)$ with the occupation time formula (2.4) of $\tilde{L}_{p}(\cdot)$ we see that

$$
\bar{L}_{p}(t)=\tilde{L}_{p}(t) \frac{1}{2} v(p) \tilde{m}(p)=\tilde{L}_{p}(t) \frac{1}{2 \tilde{S}^{\prime}(p)} \quad \text { for all } p \in(0,1) .
$$

Now applying the Itô-Tanaka formula (7.3) to $\psi_{n}(\cdot)$ and inserting (7.4), we arrive at

$$
\begin{aligned}
\psi_{n}\left(\tilde{Y}_{t}\right)-\psi_{n}\left(\tilde{Y}_{0}\right)= & \int_{0}^{t} D_{\tilde{m}} D_{\tilde{S}} \psi_{n}\left(\tilde{Y}_{u}\right) \mathrm{d} u+\int_{0}^{t} \psi_{n}^{\prime}\left(\tilde{Y}_{u}\right) \sqrt{v\left(\tilde{Y}_{u}\right)} \mathrm{d} B_{u} \\
& +\frac{1}{2} \tilde{L}_{1 / n}(t) D_{\tilde{S}} \psi_{n}\left(\frac{1}{n}\right)-\frac{1}{2} \tilde{L}_{1-1 / n}(t) D_{\tilde{S}} \psi_{n}\left(1-\frac{1}{n}\right) .
\end{aligned}
$$

Note that the Lebesgue measure of $\left\{u \leq t: \tilde{Y}_{u} \in\{0,1\}\right\}$ is equal to 0 a.s. Letting $n \rightarrow \infty$ in (7.5) completes the proof.

Proof of Theorem 2.1. Recall $\tilde{G}, D_{\tilde{m}} D_{\tilde{S}}$, and $\mathbb{H}$ from Section 5. Lemma 5.2 shows that the generator of the time-reversed process is the closure of $\tilde{G}$. Therefore, it remains to be shown that the generator of the Markov process $(\tilde{p}(t): t \geq 0)$ restricted to the set $\mathbb{H}$ coincides with $\tilde{G}$, that is, that

$$
\frac{\mathrm{E}^{p} \psi(\tilde{p}(t))-\psi(p)}{t} \rightarrow \tilde{G} \psi(p)=D_{\tilde{m}} D_{\tilde{S}} \psi(p) \quad \text { as } t \rightarrow 0
$$

holds for all $p \in[0,1]$ and every $\psi \in \mathbb{H}$.

Recall $R_{i}, r_{i}, \kappa_{i}$ for $i \in\{0,1\}$ from Section 2. Fix $\psi \in \mathbb{H}$, and note that $\psi$ is $\mathcal{C}^{2}$ in $(0,1)$. Using Itô's formula, it is straightforward to show that the convergence in (7.6) holds for every $p \in(0,1)$ if $\lambda \kappa_{i} \neq 0$ and holds for every $p \in[0,1]$ if $\lambda \kappa_{i}=0$. It remains to prove (7.6) for $i \in\{0,1\}$ if $\lambda \kappa_{i} \neq 0$. Starting at $i \in\{0,1\},(\tilde{p}(t): t \geq 0)$ evolves according to a diffusion $(\tilde{Y}(t): t \geq 0)$ which is governed by $\tilde{A}$ until the first time $t$ such that $\tilde{L}_{i}(t) \geq R_{i}$. At that time, the process restarts from an independent random point $J_{i}$ in $(0,1)$ with distribution $\left(1 / \kappa_{i}\right) w_{i}(p) \pi(p) \mathrm{d} p$. Thus,

$$
\begin{aligned}
\mathrm{E}^{i} \psi & (\tilde{p}(t))-\psi(i)-\mathrm{E}^{i}\left[\mathbf{1}_{\left\{\tilde{L}_{i}(t)<R_{i}\right\}}\left(\psi\left(\tilde{Y}_{t}\right)-\psi(i)\right)\right] \\
& =\mathrm{E}^{i}\left[\int_{0}^{\tilde{L}_{i}(t)} \mathrm{E}^{J_{i}}[\psi(\tilde{p}(t-l))-\psi(i)] r_{i} \mathrm{e}^{-r_{i} l} \mathrm{~d} l\right] \\
& =\mathrm{E}^{i}\left[\int_{0}^{\tilde{L}_{i}(t)}\left(\int_{0}^{1}(\psi(z)-\psi(i)) \frac{1}{\kappa_{i}} w_{i}(z) \pi(z) \mathrm{d} z+O(t-l)\right) r_{i} \mathrm{e}^{-r_{i} l} \mathrm{~d} l\right] \\
& =\mathrm{E}^{i}\left[1-\mathrm{e}^{\left.-r_{i} \tilde{L}_{i}(t)\right]} \int_{0}^{1}[\psi(z)-\psi(i)] \frac{1}{\kappa_{i}} w_{i}(z) \pi(z) \mathrm{d} z+O\left(t \mathrm{E}^{i} \tilde{L}_{i}(t)\right)\right. \\
& =o(t)+\mathrm{E}^{i}\left[r_{i} \tilde{L}_{i}(t)\right](-1)^{i+1} \lim _{p \rightarrow i}\left(\frac{1}{2} v \pi \psi^{\prime}\right)(p) \frac{1}{\lambda \kappa_{i}}+O\left(t \mathrm{E}^{i} \tilde{L}_{i}(t)\right)
\end{aligned}
$$

as $t \rightarrow 0$. In the last step we used the inequality $1-\mathrm{e}^{-x}-x \leq x^{2}$ for $x \geq 0$ together with Lemma 6.3 and $\psi \in \mathbb{H}$. The local time $\tilde{L}_{i}(t)$ converges to 0 a.s. as $t \rightarrow 0$. By the dominated convergence theorem, this implies that $\mathrm{E}^{i} \tilde{L}_{i}(t)$ converges to 0 as $t \rightarrow 0$. Thus, the last summand on the right-hand side of (7.7) is of order $o(t)$. Furthermore, Lemma 4.3 implies that

$$
r_{i} \lim _{p \rightarrow i}\left(v \pi \psi^{\prime}\right)(p) \frac{1}{\lambda \kappa_{i}}=\lim _{p \rightarrow i}\left(v \tilde{m} \psi^{\prime}\right)(p)=D_{\tilde{S}} \psi(i) .
$$


Next we consider the second expectation on the left-hand side of (7.7). Using Hölder's inequality, we see that

$$
\begin{aligned}
\mathrm{E}^{i}\left[\mathbf{1}_{\left\{\tilde{L}_{i}(t) \geq R_{i}\right\}}(\psi(\tilde{Y}(t))-\psi(i))\right] & =\mathrm{E}^{i}\left[\left(1-\mathrm{e}^{-r_{i} \tilde{L}_{i}(t)}\right)(\psi(\tilde{Y}(t))-\psi(i))\right] \\
& \leq \sqrt{\mathrm{E}^{i}\left[\left(\tilde{L}_{i}(t)\right)^{2}\right]} \sqrt{\mathrm{E}^{i}\left[(\psi(\tilde{Y}(t))-\psi(i))^{2}\right]} \\
& =\sqrt{o(t)} \sqrt{O(t)} \\
& =o(t),
\end{aligned}
$$

where we have applied Lemma 6.3. Thus, we obtain, from the Itô-Tanaka formula (7.1),

$$
\begin{aligned}
\mathrm{E}^{i}\left[\mathbf{1}_{\left\{\tilde{L}_{i}(t)<R_{i}\right\}}(\psi(\tilde{Y}(t))-\psi(i))\right] & =o(t)+\mathrm{E}^{i}[\psi(\tilde{Y}(t))-\psi(i)] \\
& =o(t)+t D_{\tilde{m}} D_{\tilde{S}} \psi(i)+(-1)^{i} \frac{1}{2} \mathrm{E}^{i}\left[\tilde{L}_{i}(t)\right] D_{\tilde{S}} \psi(i)
\end{aligned}
$$

as $t \rightarrow 0$. Putting (7.7), (7.8), and (7.9) together completes the proof of Theorem 2.1.

\section{Acknowledgements}

We are grateful to Tom Kurtz, Alison Etheridge, and two anonymous referees for their suggestions and comments on the manuscript.

\section{References}

[1] Barton, N. H. and Etheridge, A. M. (2004). The effect of selection on genealogies. Genetics 166, 1115-1131.

[2] Barton, N. H., Etheridge, A. M. and Sturm, A. K. (2004). Coalescence in a random background. Ann. Appl. Prob. 14, 754-785.

[3] Birkhoff, G. And Rota, G.-C. (1989). Ordinary Differential Equations, 4th edn. John Wiley, New York.

[4] Blumenthal, R. M. and Getoor, R. K. (1968). Markov Processes and Potential Theory (Pure Appl. Math. 29). Academic Press, New York.

[5] Coop, G. And Griffiths, R. C. (2004). Ancestral inference on gene trees under selection. Theoret. Pop. Biol. 66, 219-232.

[6] Donnelly, P. and Kurtz, T. G. (1999). Genealogical processes for Fleming-Viot models with selection and recombination. Ann. Appl. Prob. 9, 1091-1148.

[7] Ethier, S. N. And Kurtz, T. G. (1986). Markov Processes. John Wiley, New York.

[8] Ewens, W. J. (2004). Mathematical Population Genetics. I, 2nd edn. Springer, New York.

[9] Getoor, R. K. and Sharpe, M. J. (1981). Two results on dual excursions. In Seminar on Stochastic Processes, 1981 (Evanston, IL, 1981; Progress Prob. Statist. 1), Birkhäuser Boston, MA, pp. 31-52.

[10] Gillespie, J. H. (2000). Genetic drift in an infinite population: the pseudohitchhiking model. Genetics $\mathbf{1 5 5}$, 909-919.

[11] Itô, K. And McKean, H. P., JR. (1974). Diffusion Processes and Their Sample Paths. Springer, Berlin.

[12] Kaplan, N. L., Darden, T. and Hudson, R. R. (1988). The coalescent process in models with selection. Genetics 120, 819-829.

[13] Karatzas, I. and Shreve, S. E. (1991). Brownian Motion and Stochastic Calculus (Graduate Texts Math. 113), 2nd edn. Springer, New York.

[14] Karlin, S. and Taylor, H. M. (1981). A Second Course in Stochastic Processes. Academic Press, New York.

[15] KIM, Y. (2004). Effect of strong directional selection on weakly selected mutations at linked sites: implication for synonymous codon usage. Mol. Biol. Evol. 21, 286-294.

[16] Kingman, J. F. C. (1982). On the genealogy of large populations. In Essays in Statistical Science (J. Appl. Prob. Spec. Vol. 19A), eds J. Gani and E. J. Hannan, Applied Probability Trust, Sheffield, pp. 27-43.

[17] Kingman, J. F. C. (1982). The coalescent. Stoch. Process. Appl. 13, 235-248.

[18] Krone, S. M. and Neuhauser, C. (1997). Ancestral processes with selection. Theoret. Pop. Biol. 51, $210-237$.

[19] Mandl, P. (1968). Analytical Treatment of One-Dimensional Markov Processes. Springer, New York.

[20] McKean, H. P., JR. (1956). Elementary solutions for certain parabolic partial differential equations. Trans. Amer. Math. Soc. 82, 519-548.

[21] Mitro, J. B. (1984). Exit systems for dual Markov processes. Z. Wahrscheinlichkeitsth. 66, 259-267. 
[22] Mitro, J. B. (1984). Time reversal depending on local time. Stoch. Process. Appl. 18, 171-177.

[23] Nelson, E. (1958). The adjoint Markoff process. Duke Math. J. 25, 671-690.

[24] Neuhauser, C. and Krone, S. M. (1997). The genealogy of samples in models with selection. Genetics $\mathbf{1 4 5 ,}$ 519-534.

[25] Press, W. H., Teukolsky, S. A., Vetterling, W. T. and Flannery, B. P. (1992). Numerical Recipes in C, 2nd edn. Cambridge University Press.

[26] Rogers, L. C. G. And Williams, D. (2000). Diffusions, Markov Processes, and Martingales. Vol. 2. Cambridge University Press.

[27] Rong, R. et al. (2007). Unique mutational patterns in the envelope alpha 2 amphipathic helix and acquisition of length in gp120 hypervariable domains are associated with resistance to autologous neutralization of subtype C human immunodeficiency virus type 1. J. Virol. 81, 5658-5668.

[28] Rouzine, I. M. And Coffin, J. M. (1999). Search for the mechanism of genetic variation in the pro gene of human immunodeficiency virus. J. Virol. 73, 8167-8178.

[29] Rubin, L. G. (1987). Bacterial colonization and infection resulting from multiplication of a single organism. Rev. Infect. Diseases 9, 488-493.

[30] TAYlor, J. E. (2007). The common ancestor process for a Wright-Fisher diffusion. Electron. J. Prob. 12, 808847.

[31] Yuste, E. et al. (1999). Drastic fitness loss in human immunodeficiency virus type 1 upon serial bottleneck events. J. Virol. 73, 2745-2751. 